\title{
Modelling multiple cycles of static and dynamic recrystallisation using a fully implicit isotropic material model based on dislocation density
}

Gerhardus J. Jansen van Rensburg

\section{Abstract}

This paper presents the development and numerical implementation of a state variable based thermomechanical material model, intended for use within a fully implicit finite element formulation. Plastic hardening, thermal recovery and multiple cycles of recrystallisation can be tracked for single peak as well as multiple peak recrystallisation response. The numerical implementation of the state variable model extends on a $J_{2}$ isotropic hypo-elastoplastic modelling framework. The complete numerical implementation is presented as an Abaqus UMAT and linked subroutines. Implementation is discussed with detailed explanation of the derivation and use of various sensitivities, internal state variable management and multiple recrystallisation cycle contributions. A flow chart explaining the proposed numerical implementation is provided as well as verification on the convergence of the material subroutine. The material model is characterised using two high temperature data sets for Cobalt and Copper. The results of Finite Element Analyses using the material parameter values characterised on the Copper data set are also presented.

Keywords: Recrystallisation; Constitutive behaviour; Mechanical threshold strength; Elastoviscoplastic material; Abaqus UMAT

Gerhardus J. Jansen van Rensburg

(1) Modelling and Digital Science, Council for Scientific and Industrial Research, Pretoria, South Africa

(2) Computer Science and Applied Mathematics, University of the Witwatersrand, Johannesburg, South Africa

Contact e-mail: jjvrensburg@csir.co.za

Schalk Kok and Daniel N. Wilke

(3) Centre for Asset Integrity Management, Department of Mechanical and Aeronautical Engineering, University of Pretoria, Pretoria, South Africa

Contact e-mail: nico.wilke@up.ac.za·schalk.kok@up.ac.za

\author{
Schalk Kok Daniel N. Wilke
}

\section{Introduction}

To capture the dominant strengthening and softening mechanisms associated with microstructural changes in high temperature metal processing requires the implementation of sophisticated material models. To model material behaviour subject to recrystallisation, one option could be to link multiple model resolutions. Multi scale recrystallisation modelling strategies could involve linking a finite element code with Monte Carlo Potts [23, 25], cellular automaton (CA) [21, 43], phase field (PF) models [10, 39, 53], vertex or front-tracking $[38,54]$ as well as level set methods $[7,22]$. In terms of recrystallisation model developments linked to dislocation density based mechanical response models, Lee and Im [36] coupled a CA model to a Kocks Mecking (KM) [32, 33] type dislocation density based model formulation. Takaki et al. [49] also recently linked a multi-PF dynamic recrystallisation model to macroscopic mechanical response using $J_{2}$ flow theory. In their approach the meso-scale microstructure and large deformation elastoplastic finite element values are linked assuming an average dislocation density for each grain, evolving according to a KM model.

Our primary application of interest is to simulate industrial metal forming processes such as hot rolling [28]. Of specific interest is the ability to design a rolling schedule i.e. how many roll passes are required, and what percentage reduction is required per pass. Our material model choice should therefore be able to be integrated into a finite element environment, resulting in a simulation tool that can solve numerous roll pass schedules efficiently using standard desktop computational resources. Industrial forming processes typically do not contain plastic instabilities or localisation, therefore a material model with an embedded length scale is not required to obtain mesh independent results. Furthermore, a process such as hot rolling does not proceed till failure, therefore the material model does not require damage or failure descriptions. Therefore an isotropic continuum or mean field constitutive model is an attractive option for this application, since it is computationally efficient. The material model must however contain all the required deformation mechanicsms that are typically active during hot forming. 
The proposed model captures the dominant strengthening and softening physics associated with the microstructural changes of metallic materials. Some of the physical mechanisms associated with these microstructural changes include strain hardening, dynamic and thermal recovery as well as static and dynamic recrystallisation. As foundation for model development, the KM formulation is a popular choice in dislocation density based models. This formulation incorporates temperature and rate dependent deformation mechanisms. The hardening behaviour of a material can be modelled within this formulation via the average dislocation density or some other internal state variable (ISV). Such hardening models can even be done for alloys with multiple phases that is hot worked [17] or linked to additional evolution equations to model recrystallisation kinetics $[8,19,27,28,48]$. A continuum or mean field modelling approach to recrystallisation could further have critical recrystallisation criteria that depend explicitly on strain, strain rate or another critical value of stored energy [3, 42, 45]. If high energy grain boundaries are the dominant recrystallisation mechanism, it is also possible to base the model kinetics on the mobility of grain and subgrain boundaries with the driving force provided by the stored energy in the dislocation structure [11].

Using mean field models, macroscopic material response can be modelled as an averaged result over a representative set of spherical grains subject to discontinuous dynamic recrystallisation [6, 40]. In these models, different evolution equations for the dislocation density, stress - strain relationship and grain size evolution are considered. Each grain has a set of state variables to represent grain size and dislocation density. A grain either grows or shrinks as a result of interaction with the surrounding material, typically idealised using mean field values. During recrystallisation, new grains are nucleated using a phenomenological rate equation and hardening follows the KM theory. Chaboche-type hardening can also be used while new grains nucleate within existing material once sufficiently high energy density allows it [44]. Grain growth as a result of grain boundary energy and pinning as a result of the precipitation and dissolution of particles are also taken into account in the mean field approach of Riedel and Svoboda [44].

On the other hand, continuum models using unified sets of constitutive equations may be developed. Baron et al. [5] for example use a continuum approach to model the microstructure evolution with dynamic recrystallisation of a high strength martensitic steel. The strong dependence of the dynamic recrystallisation kinetics on the initial microstructure are taken into account during their model development. Another KM based continuum material model by Lin et al. [37] makes use of a normalised dislocation density variable coupled with evolution equations on the average grain size as well as recrystallised volume fraction. They use a set of unified viscoplastic equations to model a two roll-pass reduction schedule. The same continuum model was also recently used to model the microstructural evolution during hot cross wedge rolling [29], illustrating the continued usefulness and relevance of continuum based recrystallisation models in the finite element simulation of material processing.

In the model for static and dynamic recrystallisation validated by Brown and Bammann [8], the KM work hardening theory is again used as foundation to the constitutive formulation. Statistically necessary dislocation density plays the role of a primary stress-like ISV. The effect of geometrically necessary dislocations are also included using a stage IV stress-like work hardening variable [35]. Recrystallisation through predominantly high energy grain boundary driven kinetics is incorporated based on a continuum approximated average grain boundary mobility and driving pressure [11]. The model has the ability to represent multiple cycles of recrystallisation.

In this paper, much of the same recrystallisation theory of Chen et al. [11] as used by Brown and Bammann [8] is considered, but within a dislocation density ratio based modelling framework built in turn on Estrin's [15] constitutive model and the kinetics of the Mechanical Threshold Stress (MTS) [18] model. To the authors' knowledge, these modelling components have never been combined before in this fashion and it effectively expands the range of temperatures where the popular MTS model could be applied. In the model presented and implemented in this paper, the choice of internal state variables, evolution equations and kinetic equation is therefore different from that of Brown and Bammann [8].

Specific focus is also given in this paper to incorporating the model into a fully implicit finite element environment, which was not done by Brown and Bammann [8]. A fully implicit formulation requires the stress derivative with respect to the strain increment, and this paper is the first to provide these non-trivial derivations and implementation. The current implementation is limited to isothermal analyses, since the derivative of the stress update with respect to temperature is not yet implemented. The proposed model is used in plane strain, axisymmetric and full three dimensional finite element analyses. The finite element implementation uses effective internal state variable management and an incrementally objective Abaqus [1] user material (UMAT) framework.

The main structure of this paper is organised into five sections. First the material model framework as seen from a hypo-elastoplastic treatment of numerical plasticity is discussed in Section 2. The theory and development of the constitutive model, ignoring the effects of recrystallisation, are then covered in Section 3. Section 4 is devoted to the recrystallisation modelling approach. The model is characterised to experimental data in Section 5 and used in a finite element anal- 
ysis of a compression test in Section 6. The appendices contain a detailed numerical implementation into an Abaqus UMAT framework and the associated Fortran subroutines. While Abaqus is used in this paper, much of the subroutines may be used "as-is" in other FEA packages where Fortran user materials are possible. The detailed implementation, flow charts and analytical sensitivities may also be used to expedite implementation into a completely different format.

\section{Numerical Material Model Foundation}

The paper derives and implements a model for static and dynamic recrystallisation as in the work by Brown and Bammann [8], but within a Mechanical Threshold Stress (MTS) [18] type model framework. As in the MTS model, the foundation of our constitutive model is also based on the KM work hardening theory while the effect of geometrically necessary dislocations is included using the stage IV work hardening model of Kok et al. [35]. This introduces a second internal state variable namely the average slip plane lattice misorientation. The recrystallisation kinetics of the model is consistent with that of Brown and Bammann [8], but instead of considering stress like ISVs we base recrystallisation on the dislocation density ratio and the average slip plane lattice misorientation.

As in the incrementally objective implementation of the MTS model by Mourad et al. [41], this material model is coded into an elastic trial - radial return type algorithmic implementation user material (UMAT) for $J_{2}$ isotropic hypo-elastoplasticity. The incrementally objective Abaqus UMAT framework is attached in Appendix B. This framework is described and successfully verified against the native Abaqus implementation in Van Rensburg's PhD thesis [26]. In the user material framework a purely elastic region is assumed to enclose the origin in stress space and the total velocity gradient is additively decomposed into an elastic and plastic component [46]. All the relevant tensors are corotated within Abaqus resulting in an incrementally objective elastoplastic implementation.

In general, the components of the total strain rate tensor $\dot{\varepsilon}_{i j}$ within the model framework are additively decomposed into the elastic $\dot{\varepsilon}_{i j}^{\mathrm{e}}$ and plastic $\dot{\varepsilon}_{i j}^{\mathrm{p}}$ components

$$
\dot{\varepsilon}_{i j}=\dot{\varepsilon}_{i j}^{\mathrm{e}}+\dot{\varepsilon}_{i j}^{\mathrm{p}} .
$$

The elastic part obeys Hooke's law

$$
\dot{\varepsilon}_{i j}^{\mathrm{e}}=C_{i j k l}^{-1} \dot{\sigma}_{k l}
$$

where

$$
C_{i j k l}^{-1}=\frac{1}{2 \mu}\left(\delta_{i k} \delta_{j l}-\frac{\nu}{1+\nu} \delta_{i j} \delta_{k l}\right) .
$$

$\dot{\sigma}_{i j}$ is the time derivative of the stress tensor, $\mu$ is the shear modulus, $\nu$ is Poisson's ratio and $\delta_{i j}$ represents Kronecker's delta. The temperature dependent shear modulus is determined using the model developed by Varshni [52],

$$
\mu(T)=\mu_{r}-\frac{D_{r}}{\exp \left(T_{r} / T\right)-1},
$$

where $\mu_{r}, D_{r}$ and $T_{r}$ are reference material constants while $T$ is the absolute temperature. The model in Eq.(4) has previously been used in conjunction with the KM work hardening theory [4, 20, 35].

The plastic part of the strain rate tensor takes the form of the Lévy-von Mises equation

$$
\dot{\varepsilon}_{i j}^{\mathrm{p}}=\frac{3}{2} \frac{\dot{\alpha}}{\sigma_{\mathrm{vM}}} s_{i j},
$$

where $s_{i j}$ are the components of the deviatoric stress, $\dot{\alpha}$ is the equivalent plastic strain rate and $\sigma_{\mathrm{vM}}$ the von Mises equivalent stress. Considering plastic isotropy of the material, the effective von Mises plastic strain rate and stress are determined by

$$
\dot{\alpha}=\sqrt{\frac{2}{3} \dot{\varepsilon}_{i j}^{\mathrm{p}} \dot{\varepsilon}_{i j}^{\mathrm{p}}} \quad \text { and } \quad \sigma_{\mathrm{vM}}=\sqrt{\frac{3}{2} s_{i j} s_{i j}} .
$$

The effective yield stress is

$$
\sigma_{\mathrm{y}}=\hat{\sigma}_{a}+S_{\varepsilon}(\dot{\alpha}, T) \hat{\sigma}_{\varepsilon}
$$

where $\hat{\sigma}_{\varepsilon}$ represents the evolving thermal component of the threshold stress representing the material state. $\hat{\sigma}_{a}$ represents the athermal stress component while $S_{\varepsilon}$ is a temperature and equivalent strain rate dependent scaling function [18].

\section{Constitutive model}

We have yet to consider options for the scaling function $S_{\varepsilon}$ and the evolving thermal component that define the constitutive model within the presented framework. The scaling function $S_{\varepsilon}$ is based on the choice of kinetic equation or evolution of plastic flow. Three typical scaling function choices are the power law [31], the hyperbolic sine form [8] or a scaling function based on that of the Mechanical Threshold Stress (MTS) model [18]. In this study we consider the latter which is given by

$$
S_{\varepsilon}(\dot{\alpha}, T)=\frac{\mu}{\mu_{r}}\left[1-\left[\frac{T}{a_{0} \mu} \ln \left(\frac{\dot{\varepsilon}_{0}}{\dot{\alpha}}\right)\right]^{1 / q}\right]^{1 / p},
$$

with $a_{0}$ a convenient constant introduced here to replace $g_{0} b^{3} / k_{\mathrm{B}}$ in the MTS formulation. Here, $g_{0}$ is the normalised activation energy, $b$ is the length of the Burgers' vector and $k_{\mathrm{B}}$ is the Boltzmann constant. $\dot{\varepsilon}_{0}$ is taken as a material constant associated with the mobility of dislocations. Lastly, $p$ and $q$ are statistical 
parameters that characterise the shape of the obstacle profile [18]. $p$ is usually chosen between 0 and 1 , while $q$ is between 1 and 2 .

In the classic implementation of an MTS type model, the evolving thermal stress component $\hat{\sigma}_{\varepsilon}$ in Eq.(7) is used as an internal state dependent variable. Evolution of this variable as a result of plastic deformation is therefore required to complete the constitutive formulation. Following the model development by Estrin [15], this is achieved by introducing a stress related constant $\sigma_{0}$ at initial dislocation density $\rho_{0}$. We can now recast the formulation to a dislocation density ratio $\varrho$ internal state variable. $\varrho$ is the dislocation density $\rho$ normalised by the initial dislocation density $\rho_{0}$. The evolving thermal component of the threshold stress $\hat{\sigma}_{\varepsilon}$ is then related to the dislocation density ratio ISV by

$$
\hat{\sigma}_{\varepsilon}=\sigma_{0} \sqrt{\varrho}
$$

The constitutive formulation may now be completed by using the theory on dislocation density based modelling to evolve the dislocation density ratio as a function of plastic deformation. In the MTS model the dislocation density evolution equation is transformed so that the threshold stress is a function of itself. One such form reconcilable with the Kocks-Mecking hardening theory is the popular Voce [34] hardening law. In the dislocation density ratio based formulation presented here, the dislocation density ratio is evolved instead of the threshold stress.

\subsection{Statistically stored dislocations}

The generation and annihilation of statistically stored dislocations are included in the Kocks-Mecking evolution equation [32]. In their equation, the first term corresponds to generation rate, the rate at which dislocations become immobilised. This term is assumed inversely proportional to the mean free path a dislocation travels before being affected by impenetrable obstacles. The second term corresponds to thermally activated dynamic recovery. This term is assumed proportional to the dislocation density itself.

The hybrid theory of Estrin and Mecking [16] expands on the KM evolution equation by further assuming that the mean free path is influenced by interactions with other dislocations and subgrain boundaries due to geometrically necessary dislocations.

Including an Arrhenius type static or thermal recovery term similar to that used by Song and McDowell [47], the evolution equation for statistically stored dislocation density as used by Kok et al. [35] takes the rate form

$$
\dot{\rho}=\dot{\alpha}\left[\frac{k_{0}}{L_{g}}+\frac{k_{1}}{L_{s}}-k_{2}(\dot{\alpha}, T) \rho\right]-k_{3}(T) \rho^{r_{3}} .
$$

This evolution equation has material constants $k_{0}$ and $k_{1}$, a dynamic recovery function $k_{2}(\dot{\alpha}, T)$ and static recovery function $k_{3}(T)$ with a dislocation density dependency captured by the exponent $r_{3} . L_{g}$ is the geometric mean free path while $L_{s} \propto 1 / \sqrt{\rho}$ is the mean free path associated with dislocation interactions.

\subsection{Geometrically necessary disloca- tions}

While the statistically determined mean free path relates to the total dislocation density through $L_{s} \propto$ $1 / \sqrt{\rho}$, the geometrically determined mean free path $L_{g}$ relates to the average slip plane lattice incompatibility $\lambda[2,35]$. The relationship between the geometrically determined mean free path and average lattice incompatibility can be modelled in the same way as the statistical mean free path by $L_{g} \propto 1 / \sqrt{\lambda}$. Considering the net dislocations are arranged in a linear fashion however, Acharya and Beaudoin [2] used the relationship $L_{g} \propto 1 / \lambda$, leading more generally to the empirical statement by Kok et al. [35]

$$
L_{g} \propto\left(\frac{1}{\lambda}\right)^{r_{g}}
$$

where $1 / 2 \leq r_{g} \leq 1$ is a parameter. Kok et al. [35] further observed that the evolution of the average slip plane lattice incompatibility is inversely proportional to the grain size $d_{\mathrm{x}}$. Using the proportionality constant $C_{\lambda}$ for a specific grain size, an evolution equation for this parameter is simply

$$
\dot{\lambda}=\dot{\alpha} C_{\lambda} .
$$

\subsection{Two state variable model}

The average slip plane lattice incompatibility $\lambda$ is taken as one of the two ISVs needed to complete the formulation. Considering the dislocation density ratio $\varrho=\rho / \rho_{0}$ in Eq.(9) as the other ISV, an evolution equation for this variable is needed. This is achieved by replacing $\rho$ in Eq.(10) by $\rho_{0} \varrho$ and taking Eq.(11) into account. The result of this substitution and grouping of constants give

$$
\dot{\varrho}=\dot{\alpha}\left(C_{0} \lambda^{r_{g}}+C_{1} \sqrt{\varrho}-C_{2}(\dot{\alpha}, T) \varrho\right)-C_{3}(T) \varrho^{r_{3}},
$$

where $C_{0}=k_{0} / \rho_{0}$ and $C_{1}=k_{1} / \sqrt{\rho_{0}}$ are now constants associated with the storage terms. For the dynamic recovery $C_{2}(\dot{\alpha}, T)$ in Eq.(13), we consider the form proposed in the MTS model [18]. An analogue of the temperature and rate dependent form used in the MTS model, within a dislocation density ratio formulation is

$$
C_{2}(\dot{\alpha}, T)=C_{20} \exp \left[-\frac{T}{a_{02} \mu} \ln \left(\frac{\dot{\alpha}}{\dot{\varepsilon}_{02}}\right)\right],
$$

with material constants $C_{20}, \dot{\varepsilon}_{02}$ and $a_{02}$. As in Eq. (8), $a_{02}$ is again a convenient replacement for $g_{02} b^{3} / k_{\mathrm{B}}$ 
where $g_{02}$ represents the normalised activation energy for dislocation climb. Static thermal recovery is modelled by an expression similar to the one used by Song and McDowell [47]. An Arrhenius equation for $C_{3}(T)$ is used implying

$$
C_{3}(T)=C_{30} \exp \left(-\frac{a_{03}}{T}\right),
$$

where $C_{30}$ is a constant and $a_{03}$ is associated with the activation energy for self diffusion. Apart from the direct effects on the dislocation density ratio ISV and the evolution of the average lattice slip plane incompatibility ISV, recrystallisation is also taken into account in Section 4.

\section{Recrystallisation}

From the work by Cahn and Hagel [9], the recrystallised volume fraction growth rate is modelled using

$$
\dot{f}_{\mathrm{x}}=A_{\mathrm{x}} v_{\mathrm{x}}
$$

where $A_{\mathrm{x}}$ is the interfacial area between recrystallised and unrecrystallised regions. This is multiplied by the average velocity of the interface sweeping through the unrecrystallised region $v_{\mathrm{x}}$. The rate of interface migration is expressed using the driving pressure $P$ for boundaries with a specific energy and mobility $M$ [13]

$$
v_{\mathrm{x}}=M P .
$$

As the material deforms, the average misorientation angle $\bar{\theta}$ across the geometrically necessary subgrain boundaries increase, which in turn increases the mobility of the boundaries. Chen et al. [11] used the empirical form

$$
\bar{M}=M_{0} \exp \left(-\frac{Q_{M}}{R T}\right)\left[1-\exp \left(-C_{M}\left(\frac{\bar{\theta}}{\theta_{m}}\right)^{r_{M}}\right)\right]
$$

to express the average subgrain boundary mobility $\bar{M}$ in terms of the average misorientation angle $\bar{\theta}$ for high energy boundaries (typically $\bar{\theta} \geq \theta_{m}=15^{\circ}$ ). In Eq.(18), $M_{0}, C_{M}$ and the exponent $r_{M}$ are constants. $\theta_{m}$ is the misorientation angle associated with a high angle boundary while $R$ is the universal gas constant and $Q_{M}$ the activation energy for grain boundary mobility.

Brown and Bammann [8] replaced the misorientation angle ratio with a stress like variable related to the mean free path of geometrically necessary dislocations. This stress like variable is used as internal state variable in their model to capture geometric effects. In our implementation, the equivalent ISV is the average slip plane lattice misorientation $\lambda$ introduced in Eq.(11).

Chen et al. [11] observed the relationship between the effective subgrain diameter $\bar{d}_{\mathrm{x}}$, misorientation $\bar{\theta}$ and
Burger's vector length $b$ to be

$$
\bar{d}_{\mathrm{x}} \bar{\theta} \propto b .
$$

The average distance between geometrically necessary dislocations is assumed mainly as a result of subgrain boundaries. The mean free path of geometrically necessary dislocations is therefore proportional to the mean subgrain boundary diameter $L_{g} \propto \bar{d}_{\mathrm{x}}$. Using Eq.(11), the relationship between the average misorientation angle and the average slip plane lattice misorientation is $\bar{\theta} \propto \lambda^{r_{g}}$. The misorientation angle ratio in Eq.(18) is now replaced using this proportionality. The average subgrain boundary mobility is therefore modelled using

$$
\bar{M}=M_{0} \exp \left(-\frac{Q_{M}}{R T}\right)\left[1-\exp \left(-C_{M \theta} \lambda^{r_{M \theta}}\right)\right] .
$$

The constant $C_{M \theta}$ and exponent $r_{M \theta}$ values now differ from the original formulation to accommodate the various proportionalities.

The driving force for the motion of the geometrically necessary boundaries is the stored energy in the dislocation structure. According to Humphreys and Hatherley [24], the pressure driving the subgrain boundary growth can in it's simplest form be expressed as $P=$ $\mu b^{2} \rho / 2$. This assumes that the effect of the boundary energy on the driving force is negligible. This pressure due to the dislocation density ratio ISV is given by

$$
P=\frac{1}{2} \mu b^{2} \rho_{0} \varrho .
$$

Considering multiple recrystallisation cycles, a volume fraction $f_{\mathrm{x}_{i}}$ represents the material volume fraction that has undergone $i$ cycles of recrystallisation. Similar to the model of Brown and Bammann [8], $f_{\mathrm{x}_{i}}-f_{\mathrm{x}_{i+1}}$ represents the total volume fraction of material that has been recrystallised $i+1$ times. The original unrecrystallised material has a volume fraction $f_{\mathrm{x}_{0}}=1$ (recrystallised $i=0$ times).

Each volume fraction $f_{\mathrm{x}_{i}}-f_{\mathrm{x}_{i+1}}$ has it's own set of internal state variables $\varrho_{\mathrm{x}_{i}}$ and $\lambda_{\mathrm{x}_{i}}$. The grain boundary interfacial area between the volume fractions recrystallised $i$ and $i+1$ times is further determined by [8]

$$
\begin{array}{r}
g\left(f_{\mathrm{x}_{i}}, f_{\mathrm{x}_{i+1}}\right)=f_{\mathrm{x}_{i}}\left(\frac{f_{\mathrm{x}_{i+1}}}{f_{\mathrm{x}_{i}}}\right)^{r_{\mathrm{Rx} a}}\left(1-\frac{f_{\mathrm{x}_{i+1}}}{f_{\mathrm{x}_{i}}}\right)^{r_{\mathrm{Rx} b}} \times \\
\left(1+C_{\mathrm{Rx} c}\left(1-f_{\mathrm{x}_{i}}\right)\right),
\end{array}
$$

with $A_{\mathrm{x}}\left(f_{\mathrm{x}_{i}}, f_{\mathrm{x}_{i+1}}\right) \propto g\left(f_{\mathrm{x}_{i}}, f_{\mathrm{x}_{i+1}}\right) . \quad r_{\mathrm{Rx} a}$ and $r_{\mathrm{Rx} b}$ are exponents used in the empirical relation of the interfacial grain boundary area while $C_{\mathrm{Rxc}}$ is a constant.

Including all of the above mentioned into a single expression, the rate of recrystallisation in Eq.(16) is rewritten for the volume fraction recrystallised $i+1$ times

$$
\dot{f}_{\mathrm{x}_{i+1}}=\varrho_{\mathrm{x}_{i}} C_{\mathrm{Rx} 0} C_{\mathrm{Rx} T}(T) C_{\mathrm{Rx} \lambda}\left(\lambda_{\mathrm{x}_{i}}\right) g\left(f_{\mathrm{x}_{i}}, f_{\mathrm{x}_{i+1}}\right) .
$$


The function is rewritten so that $C_{\mathrm{Rx} 0}$ effectively contains all the pre-exponential constants. $C_{\mathrm{Rx} T}(T)$ similarly contains the temperature dependence lumped in a single function

$$
C_{\mathrm{Rx} T}(T)=\mu(T) \exp \left(-\frac{a_{0 \mathrm{Rx}}}{T}\right),
$$

with $a_{0 \mathrm{Rx}}$ a grouping similar to the one in Eq.(15). The function $C_{\mathrm{Rx} \lambda}(\lambda)$ contains the geometric effects in the rewritten function

$$
C_{\mathrm{Rx} \lambda}(\lambda)=1-\exp \left(-C_{\mathrm{Rx} \lambda 0} \lambda^{r_{\mathrm{Rx} \lambda}}\right),
$$

where $C_{\mathrm{Rx} \lambda 0}$ and $r_{\mathrm{Rx} \lambda}$ replaces the constant $C_{M \theta}$ and exponent $r_{M \theta}$ in Eq.(20) for subscript consistency.

\subsection{Internal state variable evolution}

In the absence of recrystallisation, the ISVs evolve according to Eqs.(12) and (13). Given a time increment $\delta t$, the first $\left(f_{\mathrm{x}_{1}}\right)$ and second $\left(f_{\mathrm{x}_{2}}\right)$ volume fractions can both progress, meaning region $f_{\mathrm{x}_{1}}-f_{\mathrm{x}_{2}}$ will increase by $\delta f_{\mathrm{x}_{1}}$ and decrease by $\delta f_{\mathrm{x}_{2}}$. Assuming recrystallisation removes the dislocation structure, the dislocation density ratio within a newly recrystallised portion $\delta f_{\mathrm{x}_{1}}$ should be reinitialised. If the region $f_{\mathrm{x}_{1}}(t)-f_{\mathrm{x}_{2}}(t)-\delta f_{\mathrm{x}_{2}}$ hardens or recovers in the absence of recrystallisation, the dislocation density ratio at $t+\delta t$ is given by

$$
\begin{gathered}
\left\{\varrho_{\mathrm{x}_{1}}\right\}_{f_{\mathrm{x}_{1}}(t)-f_{\mathrm{x}_{2}}(t)-\delta f_{\mathrm{x}_{2}}}^{t+\delta t} \approx\left\{\varrho_{\mathrm{x}_{1}}\right\}_{f_{\mathrm{x}_{1}}(t)-f_{\mathrm{x}_{2}}(t)}^{t}+ \\
\left(\dot{\alpha}\left(C_{0} \lambda_{\mathrm{x}_{1}}^{r_{g}}+C_{1} \sqrt{\varrho_{\mathrm{x}_{1}}}-C_{2}(\dot{\alpha}, T) \varrho_{\mathrm{x}_{1}}\right)-C_{3}(T) \varrho_{\mathrm{x}_{1}}^{r_{3}}\right) \delta t .
\end{gathered}
$$

Applying the rule of mixtures gives

$$
\begin{aligned}
& \left\{\varrho_{\mathrm{x}_{1}}\right\}_{f_{\mathrm{x}_{1}}(t+\delta t)-f_{\mathrm{x}_{2}}(t+\delta t)}^{t+\delta t}= \\
& \frac{f_{\mathrm{x}_{1}}(t)-f_{\mathrm{x}_{2}}(t)-\delta f_{\mathrm{x}_{2}}}{f_{\mathrm{x}_{1}}(t)-f_{\mathrm{x}_{2}}(t)+\delta f_{\mathrm{x}_{1}}-\delta f_{\mathrm{x}_{2}}}\left\{\varrho_{\mathrm{x}_{1}}\right\}_{f_{\mathrm{x}_{1}}(t)-f_{\mathrm{x}_{2}}(t)-\delta f_{\mathrm{x}_{2}}}^{t+\delta t}
\end{aligned}
$$

Substituting Eq.(26) into Eq.(27), the general form of the dislocation density ratio evolution can be rewritten by taking the limit $\delta t \rightarrow 0$ and substituting $i=1$ in the same way as done by Brown and Bammann [8]. In the event of recrystallisation, the rate form of the dislocation density ratio evolution equation in Eq.(13) is replaced by

$$
\begin{array}{r}
\dot{\varrho}_{\mathrm{x}_{i}}=\dot{\alpha}\left(C_{0} \lambda_{\mathrm{x}_{i}}^{r_{g}}+C_{1} \sqrt{\varrho_{\mathrm{x}_{i}}}-C_{2}(\dot{\alpha}, T) \varrho_{\mathrm{x}_{i}}\right)- \\
C_{3}(T) \varrho_{\mathrm{x}_{i}}^{r_{3}}-\frac{\dot{f}_{\mathrm{x}_{i}}}{f_{\mathrm{x}_{i}}-f_{\mathrm{x}_{i+1}}} \varrho_{\mathrm{x}_{i}}
\end{array}
$$

for the dislocation density ratio variable associated with the total volume fraction $f_{\mathrm{x}_{i}}-f_{\mathrm{x}_{i+1}}$. Similarly for the average slip plane lattice incompatibility ISV, we have

$$
\dot{\lambda}_{\mathrm{x}_{i}}=\dot{\alpha} C_{\lambda}-\frac{\dot{f}_{\mathrm{x}_{i}}}{f_{\mathrm{x}_{i}}-f_{\mathrm{x}_{i+1}}} \lambda_{\mathrm{x}_{i}} .
$$

In the presence of recrystallisation, the equivalent threshold stress of Eq.(9) is calculated from the average dislocation density ratio

$$
\bar{\varrho}=\sum_{i=0}^{n_{\mathrm{x}}-1} \varrho_{\mathrm{x}_{i}}\left(f_{\mathrm{x}_{i}}-f_{\mathrm{x}_{i+1}}\right),
$$

where $n_{\mathrm{x}}$ is the total number of active recrystallisation cycles.

A detailed numerical implementation and Fortran subroutines for use in Abaqus or other FEA software with similar user material ability are given in the appendices. The model is now characterised to experimental material response data. In the following section the model is characterised to Cobalt data and various aspects of the model are discussed using the characterised model. The material is modelled using a one dimensional material model that calls the isotropic hardening subroutine in Appendix C. To test the gradients and numerical implementation of the isotropic hardening model with recrystallisation, a single increment is also covered in detail before using the model within an FEA environment in Section 6.

\section{Model calibration and verifi- cation}

The model outlined and implemented into an isotropic UMAT framework is now characterised to data where dynamic recrystallisation is prevalent. This data set is digitised from a paper on aspects of dynamic recrystallisation in Cobalt at high temperatures by Kapoor et al. [30]. In their study they used $5 \mathrm{~mm}$ diameter by $10 \mathrm{~mm}$ cylindrical test specimens. The wrought Cobalt rod these specimens were taken from had a chemical composition of $0.05 \% \mathrm{Ni}, 0.015 \% \mathrm{Fe}, 0.005 \% \mathrm{Cu}, 0.03 \%$ $\mathrm{C}$ and $99.95 \% \mathrm{Co}$ in weight percentages. The material had an as-received average grain size of $10 \mu \mathrm{m}$. The compression tests were done between $600^{\circ} \mathrm{C}$ and $900^{\circ} \mathrm{C}$. They used a time varying ram speed so that a constant true strain rate was applied. Kapoor et al. [30] used Aluminium push rods and hexagonal Boron Nitrate as lubricant while the test chamber was flushed with Argon to prevent excessive oxidation at higher temperatures.

From displacement and load cell data they determined logarithmic true strain and true stress following a volume preserving area assumption. The combined sample and machine stiffness was used to remove the elastic strain component from the total strain. For our purposes the data was obtained by digitising values observed in their figures of true stress as a function of true plastic strain.

The stress vs strain responses for Cobalt at $600^{\circ} \mathrm{C}$, $700^{\circ} \mathrm{C}, 750^{\circ} \mathrm{C}, 800^{\circ} \mathrm{C}, 900^{\circ} \mathrm{C}$ and $950^{\circ} \mathrm{C}$ were extracted from the paper for strain rates of $10^{0} \mathrm{~s}^{-1}, 10^{-1} \mathrm{~s}^{-1}$ and 
$10^{-2} \mathrm{~s}^{-1}$. The set of preselected tunable material parameter values are determined using a penalised downhill simplex method. Initial parameter estimates are extracted from literature values and linear regression on transformed experimental data using the Arrhenius exponential form of the various temperature dependencies [26]. The material model parameters of the two ISV model are first determined ignoring the softening part of the experimental data curves. These parameter values are then fixed while tuning the material parameters associated with recrystallisation.

The material parameter values that result in the fit displayed in Figure 1 are as follow:

- The elastic properties using the shear model relationship in Eq.(4) are $\mu_{r}=81815 \mathrm{MPa}, D_{r}=$ $6519 \mathrm{MPa}, T_{r}=200 \mathrm{~K}$ and a Poisson's ratio of $\nu=$ 0.31 .

- The temperature and rate dependent scaling function in Eq.(8) is modelled with $a_{0 \varepsilon}=1.924$ $\mathrm{K} / \mathrm{MPa}, p_{\varepsilon}=2 / 3, q_{\varepsilon}=3 / 2$ and $\dot{\varepsilon}_{0 \varepsilon}=10^{7} \mathrm{~s}^{-1}$.

- The athermal yield stress component and reference stress values using Eq.(7) are $\hat{\sigma}_{a}=0 \mathrm{MPa}$ and $\sigma_{0}=83.7 \mathrm{MPa}$.

- The average slip plane lattice incompatibility is affected by a constant that can be calibrated in both Eqs.(25) and (28) where it plays a role and so the evolution of $\lambda$ according to Eq.(29) is simply modelled using $C_{\lambda}=1$.

- The parameters associated with the evolution of the dislocation density ratio in Eq.(28) are $C_{0}=$ 584.64, $r_{g}=1, C_{1}=156.61, C_{20}=7.566, a_{02}=$ $0.496 \mathrm{~K} / \mathrm{MPa}, \dot{\varepsilon}_{02}=10^{10} \mathrm{~s}^{-1}, C_{30}=12121.95 \mathrm{~s}^{-1}$, $a_{03}=39274.9 \mathrm{~K}$ and $r_{3}=7.346$.

- The recrystallisation parameters are finally $C_{\mathrm{Rx} 0}=1562.08 \mathrm{~s}^{-1}$ for the pre-exponential constant in Eq.(23), $a_{0 \mathrm{Rx}}=21049.20 \mathrm{~K}$ in Eq.(24) with $C_{\mathrm{Rx} \lambda 0}=8.442$ and $r_{\mathrm{Rx} \lambda}=2.321$ in Eq.(25).

- The equivalent interfacial subgrain boundary area function in Eq.(22) is modelled using $r_{\mathrm{Rx} a}=$ 0.0797, $r_{\mathrm{Rx} b}=1.339$ and $C_{\mathrm{Rx} c}=19.415$.

The recrystallised volume fractions and calculated equivalent dislocation densities are presented in Figure 2. Multiple recrystallised volume fractions are visible in Figure 2(a) as well as a shift of ISVs on two occasions to save memory. As is evident these shifts are enforced once $f_{\mathrm{x}_{1}}>0.999$, as discussed in Section A.1.

The contribution of each recrystallised volume fraction dislocation density ratio $\varrho_{\mathrm{x}_{i}}$ to the equivalent dislocation density ratio $\bar{\varrho}$ according to Eq.(30) is demonstrated in Figure 2(b). In this specific example, the equivalent dislocation density ratio and therefore the
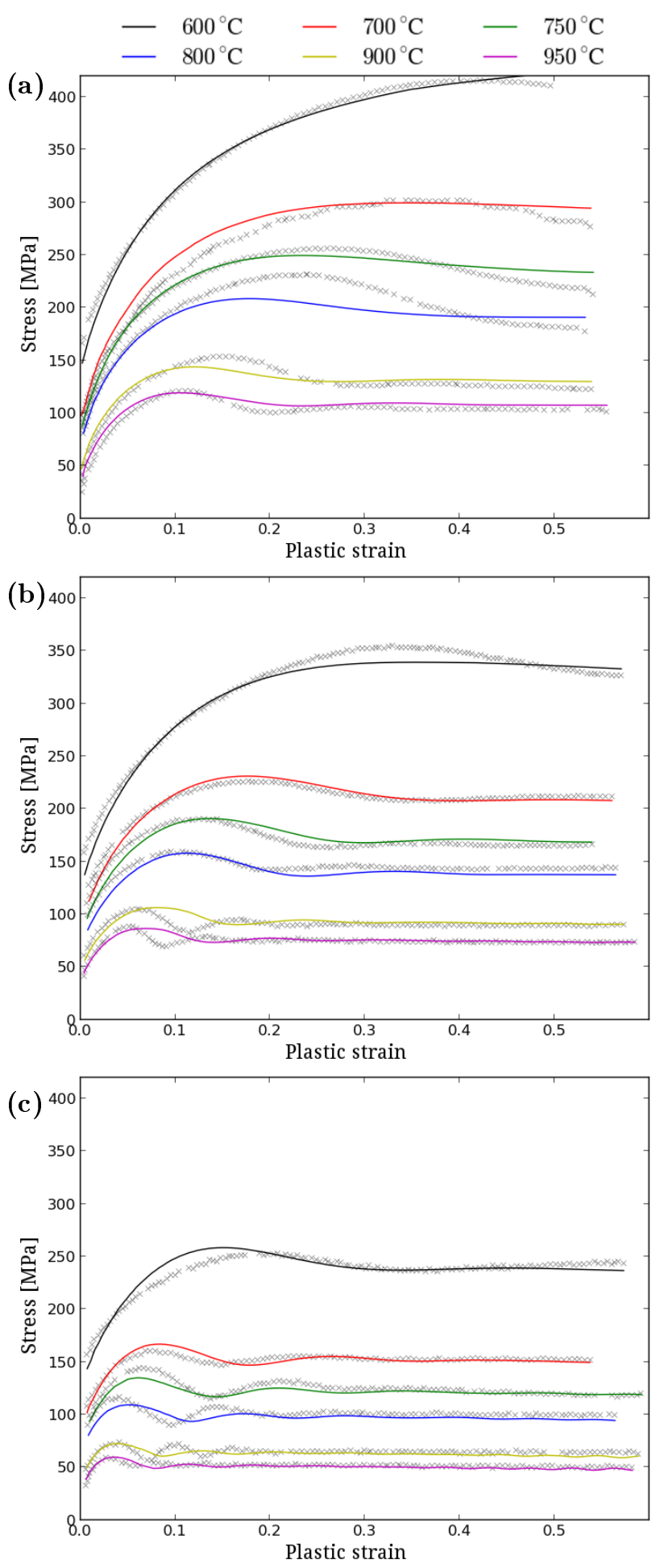

Figure 1: Numerical model (solid line) calibrated to the true stress vs. true plastic strain data for Co at different temperatures and strain rates of (a) $1 \mathrm{~s}^{-1}$, (b) $0.1 \mathrm{~s}^{-1}$ and (c) $0.001 \mathrm{~s}^{-1}$ from Kapoor et al. [30]. 
equivalent stress has a multiple peak response that approaches a steady state solution. This type of response is also visible in some of the digitised experimental data.

\subsection{Code Verification}

(a)
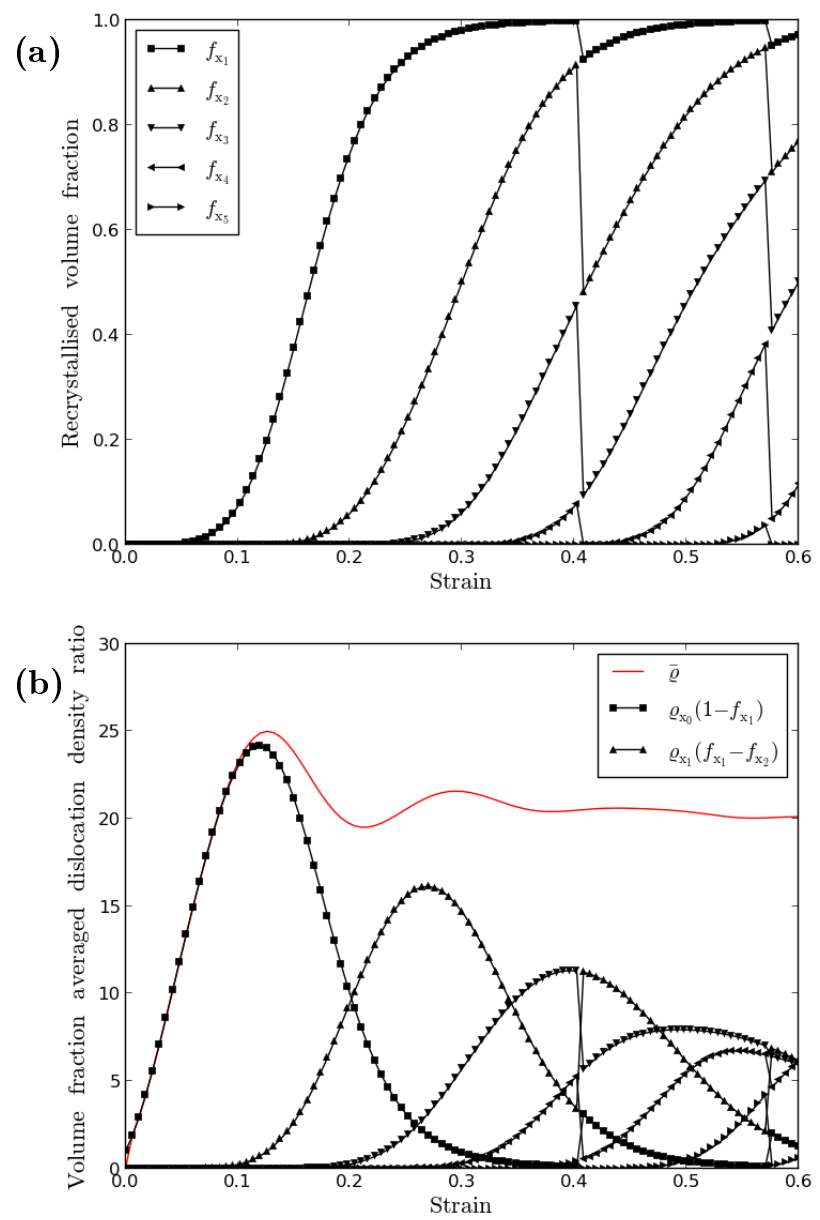

Figure 2: (a) Recrystallised volume fractions and (b) volume fraction averaged dislocation density ratio using the recrystallisation model calibrated to the cobalt dynamic recrystallisation data when modelled at $850^{\circ} \mathrm{C}$ and a strain rate of $0.1 \mathrm{~s}^{-1}$.
Using the material properties determined on the Cobalt experimental data, the code is verified by inspecting the convergence of an increment where multiple volume fractions are active. In this test, 20 increments of $\delta \varepsilon=0.01$ and $\delta t=0.1 \mathrm{~s}\left(\dot{\alpha}=0.1 \mathrm{~s}^{-1}\right)$ are analysed at $850^{\circ} \mathrm{C}$. This corresponds to a total strain of 0.2 in Figure 2(a) with at least three contributing volume fractions (original and two waves of recrystallisation). The convergence and results obtained during increment 20 is covered in detail in this subsection.

The values of the residual equation, analytical gradients as well as the estimated equivalent plastic strain increment are reported for each iteration. Components and sensitivities for the various nested solution loops are also reported for the final iteration. Forward finite difference estimates of various sensitivities are also calculated within the one dimensional test environment by perturbing the estimated plastic strains or relevant values by $10^{-8}$.

The converged yield stress and internal state variable values at the end of the $19^{t h}$ and $20^{t h}$ increment are presented in Table 1. At the end of the $19^{\text {th }}$ increment, i.e. $\varepsilon=0.19$, the yield stress is $111.967 \mathrm{MPa}$ as a result of a volume fraction averaged dislocation density $\bar{\varrho}=20.174$. Three recrystallised volume fractions are above the coded minimum of interest (0.001) namely $f_{\mathrm{x}_{1}}=0.7176, f_{\mathrm{x}_{2}}=0.08746$ and $f_{\mathrm{x}_{3}}=0.00195$ and therefore actively contribute to the stress response.

The recrystallised volume fractions grow to $f_{\mathrm{x}_{1}}=$ $0.76977, f_{\mathrm{x}_{2}}=0.11641$ and $f_{\mathrm{x}_{3}}=0.00351$ during the increment. The internal state variable values at the end of the increment are $\varrho_{\mathrm{x}_{0}}=38.533$ and $\lambda_{\mathrm{x}_{0}}=$ 0.19912 for the unrecrystallised volume fraction while $\varrho_{\mathrm{x}_{1}}=15.555$ and $\lambda_{\mathrm{x}_{1}}=0.05884, \varrho_{\mathrm{x}_{2}}=7.4652$ and $\lambda_{\mathrm{x}_{2}}=0.03014$ as well as $\varrho_{\mathrm{x}_{3}}=3.1301$ and $\lambda_{\mathrm{x}_{3}}=$ 0.01453 . The incremental update results in a yield stress of $111.168 \mathrm{MPa}$ attributed to the volume fraction averaged dislocation density ratio $\bar{\varrho}=19.8876$. This volume fraction averaged dislocation density ratio value corresponds to the value of the red line at 0.2 strain in Figure 2(b).

The solution to the $20^{\text {th }}$ increment is obtained to within a tolerance of $10^{-8}$ in five iterations as illustrated in Table 2. One additional iteration is performed resulting in a residual value of $1.42 \times 10^{-13}$. There is a quadratic trend in the convergence rate when iterations 4 and 5 are compared while the residual value 
Table 1: Converged values of the yield stress and internal state variables at the end of increment 19 and 20 used to verify the numerical implementation of the material model.

\begin{tabular}{|c|c|c|c|c||c|c|c|c|c|}
\hline Increment & Yield [MPa] & $\bar{\alpha}$ & $\bar{\varrho}$ & $\bar{\lambda}$ & Fraction & $\alpha_{\mathrm{x}_{i}}$ & $\varrho_{\mathrm{x}_{i}}$ & $\lambda_{\mathrm{x}_{i}}$ & $f_{\mathrm{x}_{i+1}}$ \\
\hline \hline 19 & 111.967 & 0.09326 & 20.1738 & 0.08955 & 0 & 0.18912 & 37.844 & 0.18912 & 0.71755 \\
\hline & & & & & 1 & 0.05905 & 14.127 & 0.05354 & 0.08746 \\
\hline & & & & & 2 & 0.03055 & 6.7714 & 0.02786 & 0.00195 \\
\hline & & & & & 3 & 0.01540 & 2.4810 & 0.01117 & 0.00010 \\
\hline \hline 20 & 111.168 & 0.09213 & 19.8876 & 0.08774 & 0 & 0.19912 & 38.533 & 0.19912 & 0.76977 \\
\hline & & & & & 1 & 0.06505 & 15.555 & 0.05884 & 0.11641 \\
\hline & & & & & 2 & 0.03296 & 7.4652 & 0.03014 & 0.00351 \\
\hline & & & & & 3 & 0.01857 & 3.1301 & 0.01453 & 0.00011 \\
\hline
\end{tabular}

Table 2: Test on the convergence of the equivalent plastic strain increment using the FISOTROPIC subroutine in Appendix C to indicate the sensitivity using Eq.(50).

\begin{tabular}{|c|c|c|c||c|c|}
\hline Iteration & Residual & $\begin{array}{c}\text { Approximate } \\
\text { Sensitivity }\end{array}$ & $\begin{array}{c}\text { Finite } \\
\text { Difference }\end{array}$ & $\begin{array}{c}\text { Plastic } \\
\text { Increment }\end{array}$ & $\begin{array}{c}\text { Variation } \\
\text { from final }\end{array}$ \\
\hline \hline 0 & 1379.36 & $2.638 \mathrm{E} 8$ & $1.844 \mathrm{E} 8$ & $1.000 \mathrm{E}-8$ & $1.001 \mathrm{E}-2$ \\
\hline 1 & 1300.63 & 6929.35 & 6925.62 & $4.704 \mathrm{E}-4$ & $9.535 \mathrm{E}-3$ \\
\hline 2 & 1223.36 & 885.011 & 891.755 & $9.623 \mathrm{E}-3$ & $3.837 \mathrm{E}-4$ \\
\hline 3 & 48.9727 & 871.341 & 878.260 & $1.001 \mathrm{E}-2$ & $1.79 \mathrm{E}-10$ \\
\hline 4 & $2.280 \mathrm{E}-5$ & 871.341 & 878.260 & $1.001 \mathrm{E}-2$ & $9.71 \mathrm{E}-15$ \\
\hline 5 & $1.239 \mathrm{E}-9$ & 871.341 & 878.260 & $1.001 \mathrm{E}-2$ & $1.73 \mathrm{E}-18$ \\
\hline 6 & $1.42 \mathrm{E}-13$ & 871.341 & 878.260 & $1.001 \mathrm{E}-2$ & - \\
\hline
\end{tabular}

from $1.239 \times 10^{-9}$ in iteration 5 to $1.42 \times 10^{-13}$ in iteration 6 indicates a deviation from the ideal quadratic convergence. This is however still orders of magnitude below the desired tolerance.

A reason for the deviation from ideal quadratic convergence is illustrated by an approximate $0.7909 \%$ difference between the sensitivity using Eq.(50) and the finite difference approximation according to Table 2 . If the sensitivities are determined and implemented correctly, this difference could be partially attributed to the multiplicative accumulation of variations as a result of the nested solution loops. The important aspect illustrated in Table 2 is the satisfactory rate of convergence of the current implementation.

Different aspects of the implemented code are investigated to pinpoint the origin of the variation observed between the finite difference and approximated sensitivity. Table 4 shows a breakdown of individual components as well as the solution to the system of equations in Eq.(59). Eq.(59) is investigated here within each volume fraction loop in the code by calls to the RGET subroutine, with the implication that the finite difference component of $d f_{\mathrm{xc}} / d \delta \alpha$ can't be compared directly. Because of this reason Table 4 illustrates the sensitivity comparisons for a case where $d f_{\mathrm{x} c} / d \delta \alpha=0$ removes the last term in Eq.(58). The tabulated values are shown up to five decimal places since that is the first location where there is an observable variation between the analytical and finite difference sensitivities in this case.
The values tabulated include the solutions to $d x_{1} / d \delta \alpha$ in Eq.(63) and $d x_{2} / d \delta \alpha$ in Eq.(64) as well as the effective value of $d f_{\mathrm{x} n} / d \delta \alpha$ in Eq.(54) for all the active volume fractions considered at the end of the increment under the test condition $d f_{\mathrm{x} c} / d \delta \alpha=0$. Partial derivatives of the internal state variables $x_{1}$ and $x_{2}$ with respect to the incremental plastic strain using Eq.(60) and Eq.(62) with $C_{\lambda}=1$ are also compared.

In Table 3 the finite differences are calculated from a call to the FISOTROPIC subroutine so that for this scenario the $d f_{\mathrm{x} c} / d \delta \alpha$ contribution is included. The finite difference contributions to $d x_{1} / d \delta \alpha$ and $d f_{\mathrm{x} n} / d \delta \alpha$ in Table 3 now differs from the values in Table 4 because of the inclusion of these other sensitivities. The $f_{\mathrm{x}_{1}}$ finite difference value of $d x_{1} / d \delta \alpha$ is now 243.246 instead of 254.675 when $d f_{\mathrm{xc}} / d \delta \alpha$ was ignored while the $f_{\mathrm{x}_{0}}$ values are the same in the two tables since $d f_{\mathrm{x} c} / d \delta \alpha=0$ is true for this case with $f_{\mathrm{x} c} \equiv f_{\mathrm{x}_{0}}=1$. While the analytical and finite difference sensitivities for the first and second volume fractions are closely similar, the difference gets larger for each next volume fraction contribution.

As illustrated in Table 3 the origin of the $0.7909 \%$ difference in Table 2 is found due to the $1.3202 \%$ difference between the finite difference and approximated sensitivity of the second term of Eq.(50). There is possibly an additional sensitivity not taken into account for the volume fractions further down the line or a small numerical error gets compounded by each subsequent volume fraction contribution. Fortuitously this vari- 
Table 3: Comparison between analytical (AN) approximation and finite difference (FD) sensitivity components of the equivalent dislocation density sensitivity used in Eq.(53).

\begin{tabular}{|c|c|c|c|c|c|c|c|}
\hline \multirow{2}{*}{$\begin{array}{c}\text { Volume } \\
\text { Fraction }\end{array}$} & \multicolumn{2}{|c|}{$d x_{1} / d \delta \alpha$} & \multicolumn{2}{c|}{$d f_{\mathrm{x} n} / d \delta \alpha$} & \multicolumn{3}{c|}{ Eq.(53) } \\
\cline { 2 - 8 } & $\mathbf{A N}$ & FD & AN & FD & AN & FD & \% Difference \\
\hline \hline$f_{\mathrm{x}_{0}}$ & 155.769 & 155.769 & 0.58624 & 0.58624 & 13.2725 & 13.2725 & - \\
\hline$f_{\mathrm{x}_{1}}$ & 242.291 & 243.246 & 1.34777 & 1.35486 & 146.458 & 146.972 & 0.35034 \\
\hline$f_{\mathrm{x}_{2}}$ & 151.061 & 167.606 & 0.10966 & 0.12127 & 26.2978 & 28.1319 & 6.73934 \\
\hline$f_{\mathrm{x}_{3}}$ & 93.1513 & 121.896 & $4.896 \mathrm{E}-4$ & $6.139 \mathrm{E}-4$ & 0.65905 & 0.79292 & 18.4398 \\
\hline TOTAL & & & & & 186.688 & 189.169 & 1.32018 \\
\hline
\end{tabular}

Table 4: Comparison between analytical (AN) approximation and finite difference (FD) sensitivity components and solution to the system of equations in Eq.(59) with $d f_{\mathrm{xc}} / d \delta \alpha=0$.

\begin{tabular}{|c|c|c|c|c|c|c|}
\hline $\begin{array}{c}\text { Volume } \\
\text { Fraction }\end{array}$ & & $\partial \delta \theta_{x_{1}} / \partial \delta \alpha+\partial \delta \theta_{x_{1}} / \partial \dot{\alpha} \times 1 / \delta t$ & $\partial \delta \theta_{x_{2}} / \partial \delta \alpha$ & $d x_{1} / d \delta \alpha$ & $d x_{2} / d \delta \alpha$ & $d f_{\mathrm{x} n} / d \delta \alpha$ \\
\hline \hline$f_{\mathrm{x}_{0}}$ & $\mathbf{A N}$ & 179.24711 & 1.00000 & 155.76870 & 1.00000 & 0.58624 \\
\hline & FD & 179.24713 & 1.00000 & 155.76871 & 1.00000 & 0.58624 \\
\hline$f_{\mathrm{x}_{1}}$ & $\mathbf{A N}$ & 284.99108 & 1.00000 & 254.67535 & 0.91628 & 1.45708 \\
\hline & FD & 284.99109 & 1.00000 & 254.67536 & 0.91628 & 1.45708 \\
\hline$f_{\mathrm{x}_{2}}$ & $\mathbf{A N}$ & 269.34973 & 1.00000 & 223.02735 & 0.78806 & 0.14355 \\
\hline & FD & 269.34973 & 1.00000 & 223.02736 & 0.78804 & 0.14355 \\
\hline$f_{\mathrm{x}_{3}}$ & $\mathbf{A N}$ & 211.70035 & 1.00000 & 170.61272 & 0.68506 & $6.675 \mathrm{E}-4$ \\
\hline & FD & 211.70035 & 1.00000 & 170.61274 & 0.68506 & $6.675 \mathrm{E}-4$ \\
\hline
\end{tabular}

ation has very little effect on the desired convergence rate and a close to quadratic trend is observed to within the desired tolerance.

In Table 5, the convergence of the solution to $x_{1}, x_{2}$ and $f_{\mathrm{x} n}$ using the RGET subroutine in Appendix $\mathrm{D}$ is also illustrated. This is done to find the solution of the internal state variables associated with $f_{\mathrm{x}_{1}}$, i.e. $x_{1} \equiv$ $\varrho_{\mathrm{x}_{1}}, x_{2} \equiv \lambda_{\mathrm{x}_{1}}$ and $f_{\mathrm{x} n} \equiv f_{\mathrm{x}_{2}}$ using the final strain increment value $\delta \alpha=0.0100063$.

Convergence of the first two recrystallised volume fractions $f_{\mathrm{x}_{1}}$ and $f_{\mathrm{x}_{2}}$ are reported in Table 6 . The iterations indicate the convergence of $f_{\mathrm{x}_{1}}$ using $\delta \alpha=0.0100063$ as well as $\varrho_{\mathrm{x}_{0}}=38.533$ and $\lambda_{\mathrm{x}_{0}}=$ 0.19912 while $f_{\mathrm{x}_{2}}$ is solved using $\varrho_{\mathrm{x}_{1}}=15.555$ and $\lambda_{\mathrm{x}_{1}}=0.05884$. Comparison of the finite difference and analytical sensitivities indicate that the solution is implemented correctly following Eq.(41) with clear quadratic convergence in both tables.

From the various convergence histories and sensitivity comparisons tabulated, the subroutines implemented in Appendix $\mathrm{C}$ and $\mathrm{D}$ are considered sufficiently accurate. The model is now also characterised on experimental data for Copper and used in Abaqus to simulate compression experiments.

\section{Recrystallisation in Copper}

Tanner and McDowell [51] performed compression experiments on $99.99 \%$ pure Copper for temperatures ranging from $25^{\circ} \mathrm{C}$ to $541^{\circ} \mathrm{C}$ and constant true strain rates ranging from quasi-static $\left(0.0004 \mathrm{~s}^{-1}\right)$ to dynamic $\left(6000 \mathrm{~s}^{-1}\right)$. For strain rates at or below $1 \mathrm{~s}^{-1}$ the tests were conducted using closed loop servo hydraulic test machines while high strain rate tests were done on a split Hopkinson pressure bar. The low strain rate compression specimens had a diameter to height ratio of 1:1.5. Concentric grooves were cut into the ends of the test specimens. These grooves were filled with different lubricants depending on the rate and temperature of the experiment. The specimen sides were also coated to prevent oxidation at higher temperatures.

As in the Cobalt case presented earlier, the stress as a function of true strain was digitised from different figures in Tanner's thesis [50]. The model is now also calibrated using the digitised data points for Tanner's Copper experiments. The material parameters are again determined using initial values from literature and linear regression on Arrhenius exponential relationships. The parameter values are then again fine-tuned using a penalised downhill simplex algorithm [26]. The material parameters resulting in the fit to the Copper data in Figure 3 are:

- $\mu_{r}=43.8 \mathrm{GPa}, D_{r}=4.7 \mathrm{GPa}, T_{r}=252 \mathrm{~K}$ and $\nu=1 / 3$ for the elastic properties using the shear model relationship in Eq.(4). 
Table 5: RGET residuals and convergence for internal state variables associated with $f_{\mathrm{x}_{1}}$ using $\delta \alpha=0.0100063$

\begin{tabular}{|c||c|c||c|c||c|c|c|c|}
\hline \multicolumn{1}{|c|}{ Iteration } & \multicolumn{2}{|c|}{ Residual } & \multicolumn{2}{|c|}{ Solution } & Sensitivity & AN & FD & \% Difference \\
\hline \hline 1 & $f_{R_{1}}$ & 1.58693 & $x_{1}$ & 14.1273 & $\partial f_{R_{1}} / \partial x_{1}$ & 1.12087 & 1.12155 & 0.06065 \\
\hline & $f_{R_{2}}$ & $5.777 \mathrm{E}-3$ & $x_{2}$ & $5.354 \mathrm{E}-2$ & $\partial f_{R_{1}} / \partial x_{2}$ & -4.33533 & -4.33542 & 0.00208 \\
\hline & $\left\|\boldsymbol{f}_{R}\right\|$ & 1.58694 & $f_{x n}$ & 0.10882 & $\partial f_{R_{2}} / \partial x_{1}$ & $9.417 \mathrm{E}-6$ & $9.541 \mathrm{E}-6$ & 1.30815 \\
\hline & & & & & $\partial f_{R_{2}} / \partial x_{2}$ & 1.08475 & 1.08475 & - \\
\hline & $f_{R_{1}}$ & $1.004 \mathrm{E}-2$ & $x_{1}$ & 15.5628 & $\partial f_{R_{1}} / \partial x_{1}$ & 1.13266 & 1.13265 & 0.00088 \\
\hline & $f_{R_{2}}$ & $1.022 \mathrm{E}-5$ & $x_{2}$ & $5.885 \mathrm{E}-2$ & $\partial f_{R_{1}} / \partial x_{2}$ & -3.76881 & -3.76787 & 0.02494 \\
\hline & $\left\|\boldsymbol{f}_{R}\right\|$ & $1.004 \mathrm{E}-2$ & $f_{x n}$ & 0.11644 & $\partial f_{R_{2}} / \partial x_{1}$ & $1.290 \mathrm{E}-5$ & $1.301 \mathrm{E}-5$ & 0.84909 \\
\hline & & & & & $\partial f_{R_{2}} / \partial x_{2}$ & 1.08780 & 1.08780 & - \\
\hline \multirow{2}{*}{} & $f_{R_{1}}$ & $2.974 \mathrm{E}-7$ & $x_{1}$ & 15.5548 & $\partial f_{R_{1}} / \partial x_{1}$ & 1.13261 & 1.13243 & 0.01589 \\
\hline & $f_{R_{2}}$ & $7.92 \mathrm{E}-11$ & $x_{2}$ & $5.884 \mathrm{E}-2$ & $\partial f_{R_{1}} / \partial x_{2}$ & $1.289 \mathrm{E}-5$ & $1.301 \mathrm{E}-5$ & 0.92664 \\
\hline & $\left\|\boldsymbol{f}_{R}\right\|$ & $2.974 \mathrm{E}-7$ & $f_{x n}$ & 0.11641 & $\partial f_{R_{2}} / \partial x_{1}$ & -3.77138 & -3.77187 & 0.01299 \\
\hline & & & & & $\partial f_{R_{2}} / \partial x_{2}$ & 1.08779 & 1.08779 & - \\
\hline & $f_{R_{1}}$ & $2.22 \mathrm{E}-16$ & $x_{1}$ & 15.5548 & $\partial f_{R_{1}} / \partial x_{1}$ & 1.13261 & 1.13398 & 0.12089 \\
\hline & $f_{R_{2}}$ & $4.34 \mathrm{E}-18$ & $x_{2}$ & $5.884 \mathrm{E}-2$ & $\partial f_{R_{1}} / \partial x_{2}$ & -3.77138 & -3.77165 & 0.00716 \\
\hline & $\left\|\boldsymbol{f}_{R}\right\|$ & $2.22 \mathrm{E}-16$ & $f_{x n}$ & 0.11641 & $\partial f_{R_{2}} / \partial x_{1}$ & $1.289 \mathrm{E}-5$ & $1.301 \mathrm{E}-5$ & 0.92664 \\
\hline & & & & & $\partial f_{R_{2}} / \partial x_{2}$ & 1.08779 & 1.08779 & - \\
\hline
\end{tabular}

Table 6: Convergence of the recrystallised volume fractions $f_{\mathrm{x}_{1}}$ and $f_{\mathrm{x}_{2}}$.

\begin{tabular}{|c|c|c|c|c|c|}
\hline Volume & \multirow{2}{*}{ Fraction } & Iteration & Residual & Fraction & \multicolumn{2}{|c|}{ Sensitivity } \\
\cline { 5 - 6 } & & & Value & AN & FD \\
\hline \hline$f_{\mathrm{x}_{1}}$ & 1 & $6.828 \mathrm{E}-2$ & 0.71755 & 1.31611 & 1.31611 \\
\hline & 2 & $4.426 \mathrm{E}-4$ & 0.76943 & 1.29844 & 1.29844 \\
\hline & 3 & $2.193 \mathrm{E}-8$ & 0.76977 & 1.29831 & 1.29831 \\
\hline & 4 & $2.78 \mathrm{E}-17$ & 0.76977 & 1.29831 & 1.29831 \\
\hline$f_{\mathrm{x}_{2}}$ & 1 & $2.999 \mathrm{E}-2$ & 0.08745 & 1.03153 & 1.03153 \\
\hline & 2 & $1.276 \mathrm{E}-4$ & 0.11654 & 1.03955 & 1.03955 \\
\hline & 3 & $1.559 \mathrm{E}-9$ & 0.11641 & 1.03955 & 1.03955 \\
\hline
\end{tabular}


- $a_{0 \varepsilon}=2.1037 \mathrm{~K} / \mathrm{MPa}, p_{\varepsilon}=1, q_{\varepsilon}=2$, and $\dot{\varepsilon}_{0 \varepsilon}=10^{6} \mathrm{~s}^{-1}$ for the temperature and rate dependent scaling function in Eq.(8).

- The athermal yield stress component is $\hat{\sigma}_{a}=12.519 \mathrm{MPa}$ and reference stress is $\sigma_{0}=$ 17.295MPa using Eq.(8).

- $C_{\lambda}=1$ is used for the evolution of $\bar{\lambda}$ according to Eq.(29).

- $C_{g}=6378.74, r_{g}=0.769, C_{1}=278.87, C_{20}=$ $11.773, a_{02}=0.904 \mathrm{~K} / \mathrm{MPa}, \quad \dot{\varepsilon}_{02}=4.0112 \times$ $10^{12} \mathrm{~s}^{-1}, C_{30}=83.07 \mathrm{~s}^{-1}, a_{03}=6370.675 \mathrm{~K}$ and $r_{3}=0.8079$ for the dislocation density ratio evolution in Eq.(28).

- $C_{\mathrm{Rx} 0}=9346.62 \mathrm{~s}^{-1}$ in Eq.(23), $a_{0 \mathrm{Rx}}=17634 \mathrm{~K}$ in Eq.(24) while $C_{\mathrm{Rx} \lambda 0}=47.247$ and $r_{\mathrm{Rx} \lambda}=3.87$ in Eq.(25). $r_{\mathrm{Rx} a}=0.1424, r_{\mathrm{Rx} b}=1.7677$ and $C_{\mathrm{Rx} c}=$ 393.44 in Eq.(22).

\subsection{Finite Element Modelling}

The appended subroutines are now used in an example modelled using Abaqus 6.11 Standard [1]. The compression of a cylindrical billet is modelled subject to rollover and barrelling at $541^{\circ} \mathrm{C}$ using the parameter values characterised to give the point integration response in Figure 3. Where Tanner's experiments were lubricated do avoid rollover, the simulations in this subsection are subject to a friction contact component between the modelled test specimen and die to enforce it. Instead of replicating Tanner's ideal experimental cases using FEA, this subsection serves as an example of more complex deformation modelled using the recrystallisation model.

Compression of a billet with an initial height of $15 \mathrm{~mm}$ and diameter of $10 \mathrm{~mm}$ is simulated in Abaqus using a $3 \mathrm{D}$ and axisymmetric model. The axisymmetric problem setup is given in Figure 4 with a $5 \mathrm{~mm} \times 7.5 \mathrm{~mm}$ quarter billet and a $7 \mathrm{~mm}$ long analytical rigid line to represent the die. The first octant is modelled in the three dimensional case due to problem symmetry, i.e half of a $\pi / 2$ billet section in the all-positive Cartesian coordinate system, with boundary conditions ensuring no out of plane movement at each of the three symmetry planes.

Contact between the die and billet top as well as the outer billet surface is permitted with hard normal contact and a friction coefficient of $\mu_{\text {frict }}=0.2$. In the three dimensional case an analytical rigid master surface is used to represent the die while in the axisymmetric case a rigid line is used. In Figure 4 the rigid line is in contact with the top billet surface at the start of the simulation and is displaced so that the axisymmetric test specimen is reduced by $60 \%$. The 0.6 true strain corresponds to a displacement of $\triangle L=7.5 \times[\exp (-0.6)-1]=-3.3839 \mathrm{~mm}$. The same axial displacement is applied to a reference point in the three dimensional case while constraining any die rotation or radial displacement.

In the three dimensional case, three simulations are performed using full integration 20 noded brick elements (C3D20). In each case the computational domain is modelled using a different average element size set through a maximum allowable edge length parameter. Using a maximum allowable edge length of $0.8 \mathrm{~mm}$, the resulting mesh consists of 315 elements. Similarly, setting it to $0.5 \mathrm{~mm}$ results in 1440 elements while a $0.3 \mathrm{~mm}$ maximum edge length results in 6825 elements. In the axisymmetric case, a maximum allowable edge length of $0.3 \mathrm{~mm}$ results in 425 eight noded square elements (CAX8) and 850 six noded triangular elements (CAX6) while a slightly smaller allowable edge length was chosen for the stiffer four noded square element resulting in a mesh with $1176 \mathrm{CAX} 4$ elements. Using each of the six meshes and corresponding formulation, a $60 \%$ true reduction with displacement applied linearly over $0.6 \mathrm{~s}$ at a temperature of $541^{\circ} \mathrm{C}$ is simulated for comparison. Automatic time stepping is used with an initial and maximum allowable time step size of $0.01 \mathrm{~s}$.

Using the reaction force extracted over time and corresponding displacement history, the true stress - true strain values are determined from

$$
\varepsilon_{\text {True }}=\ln \left(L / L_{0}\right) \text { and } \sigma_{\text {True }}=(F \times L) /\left(A_{0} \times L_{0}\right),
$$

where $L$ and $F$ are the instantaneous length and force while $L_{0}$ and $A_{0}$ are the original length and nominal area. The true stress - true strain curves in all six cases are displayed in Figure 5. From this figure all six of the simulations resulted in similar response curves.

A visual comparison on the internal material state as a result of each simulation is presented in Figure 6 . The comparison is made for the equivalent dislocation density ratio ISV between values of 120 and 140. Considering the three $3 \mathrm{D}$ simulations using (b) 315, (c) 1440 and (a,d) 6825 full integration 20 noded brick elements, the solution seems to converge as a result of mesh refinement. Further also considering the axisymmetric result using (e) $425 \times \mathrm{CAX} 8$, (f) $850 \times \mathrm{CAX} 6$ and $(\mathrm{g}) 1176 \times \mathrm{CAX} 4$, the solution is seen largely unaffected by choice of element type. The main observable and localised difference in ISV contour is seen closest to the rollover contact area. Depending on the complexity of the problem, element choice should still be carefully considered given the potential pitfalls of volumetric locking in CAX4 for example.

The effect of discontinuous loading is now also illustrated using the axisymmetric mesh consisting of 425 CAX8 elements. The same $60 \%$ reduction is applied over 0.6 seconds, broken up into two 0.3 second reductions with a variable stress free inter-compression time. The simulated die displacement up to $-1.6937 \mathrm{~mm}$ 


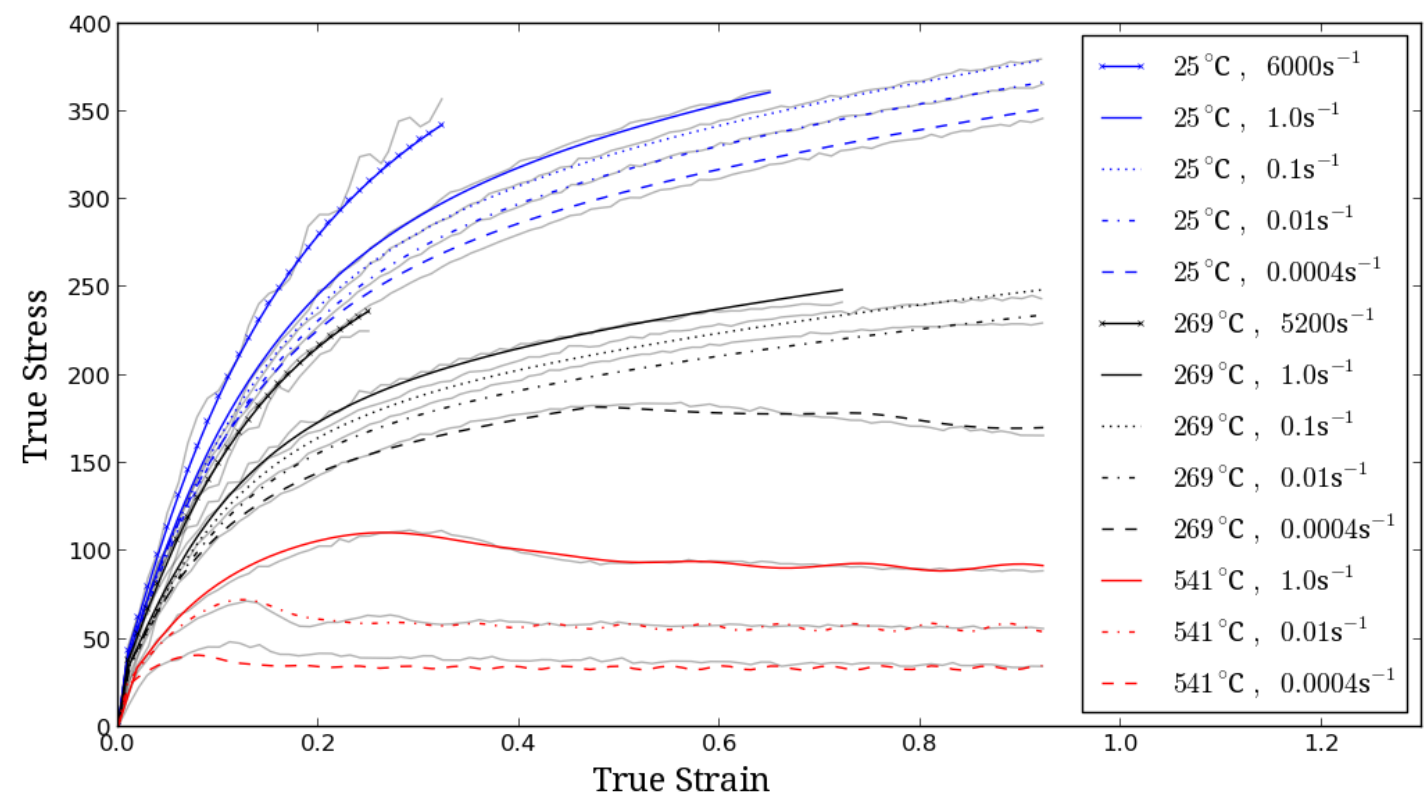

Figure 3: Numerical model (coloured lines) calibrated to Tanner and McDowell's OFHC copper data [50, 51] for different strain rates at $25^{\circ} \mathrm{C}, 269^{\circ} \mathrm{C}$ and $541^{\circ} \mathrm{C}$.

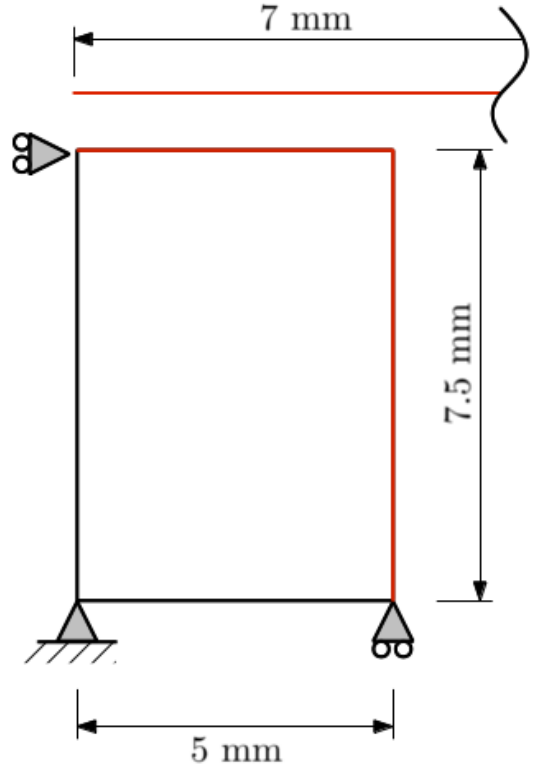

Figure 4: The axisymmetric boundary value problem setup to model a cylindrical test specimen in compression.

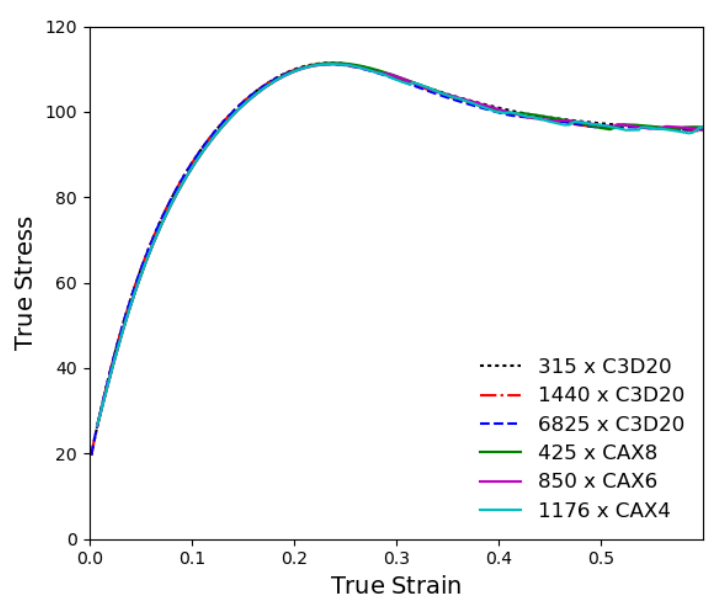

Figure 5: True stress - true strain curves as a result of the monotonic compression at $541^{\circ} \mathrm{C}$ up to $60 \%$ over 0.6 seconds. The boundary value problem is modelled using different meshes and formulations to illustrate that the same solution is obtained independent of element size and type. 


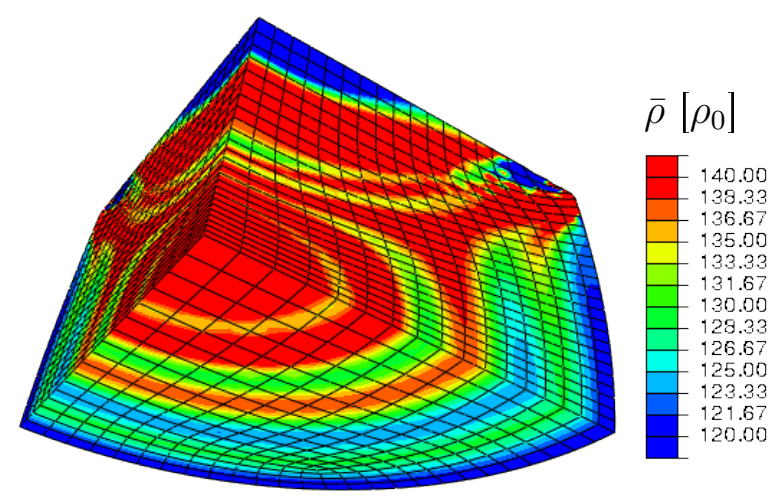

(a)

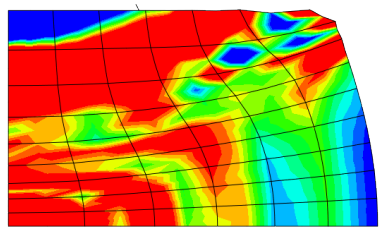

(b)

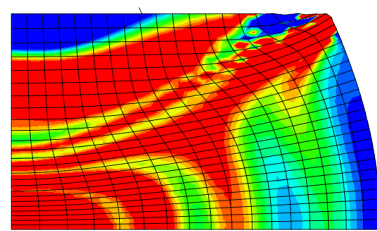

(d)

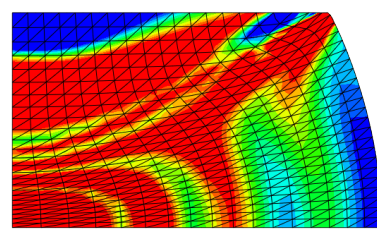

(f)

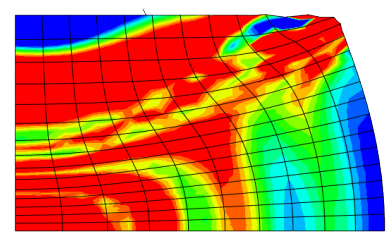

(c)

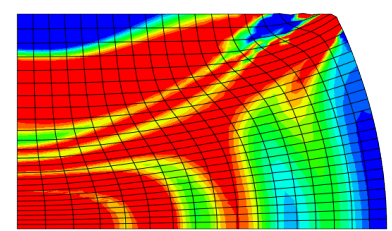

(e)

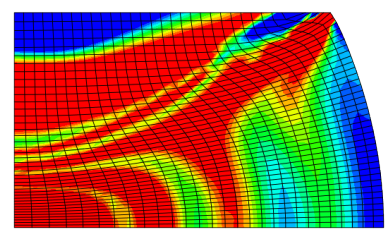

(g)
Figure 6: Equivalent dislocation density $(\bar{\rho})$ contours at $0.6 \mathrm{~s}, 541^{\circ} \mathrm{C}, 60 \%$ compression using different element types and sizes. Result using (a) $6825 \times$ full integration 20 noded brick elements (C3D20) as well as side views of the result using (b) $315 \times$ C3D20, (c) $1440 \times$ C3D20 and again (d) $6825 \times$ C3D20 to illustrate convergence within the limit of mesh refinement. The result using axisymmetric element types are given in (e) using 425 $\times \mathrm{CAX} 8,(\mathrm{f}) 850 \times \mathrm{CAX} 6$ and $(\mathrm{g}) 1176 \times \mathrm{CAX} 4$. In all these figures contours are scaled between $\bar{\rho}=120 \times \rho_{0}$ and $140 \times \rho_{0}$ where $\rho_{0}$ is the initial dislocation density, i.e. the $\bar{\varrho}=\bar{\rho} / \rho_{0}$ ISV is scaled between 120 and 140 . Values below 120 are coloured blue while values above 140 are coloured red. is first applied in $0.3 \mathrm{~s}$. The die is then displaced so that there is no contact between it and the billet for times of $1 \mathrm{~s}, 10 \mathrm{~s}$ or $60 \mathrm{~s}$. The additional $-1.6937 \mathrm{~mm}$ is applied in the $0.3 \mathrm{~s}$ to follow. The von Mises stress contours and processed true stress - true strain are plotted in Figure 7. The internal state variables related to the dislocation density as well as the volume fraction of material recrystallised at least once $\left(f_{\mathrm{x}_{1}}=\operatorname{STATEV}(7)\right)$ and at least twice $\left(f_{\mathrm{x}_{2}}=\operatorname{STATEV}(11)\right)$ are also illustrated in Figure 8.

\section{Conclusions}

The material model developed and implemented in this paper includes mechanisms for strain hardening, dynamic and thermal recovery as well as recrystallisation. The material model has the ability to simulate single and multiple peak responses due to recrystallisation and can represent a large range of temperature and strain rate responses that can now be modelled using finite element analysis in a mean field manner.

The complete numerical implementation was derived and explained in detail. Comparison of the finite difference sensitivities and those derived analytically illustrate a satisfactory agreement and close to quadratic convergence for the test increment used during this verification. Given the detailed description and subroutines provided, it is the authors' hope that the material model implementation will be useful to others for use in Abaqus [1] or another finite element package that makes use of a similar material subroutine structure. To the best of the authors' knowledge the same material subroutines can for example be used in the open source finite element solvers Calculix [12] and Code Aster [14].

As illustrated in this paper, the mean field recrystallisation model implemented has the ability to model a wide range of metal response undergoing isotropic plastic deformation. This was illustrated using high temperature (fcc) Cobalt and Copper data.

Using the model implemented, recrystallisation and internal material state can now be studied within an FEA environment using the appended subroutines. If experimentalists also examine cross sections at the end of the experiment, the modelling may also be validated, improved or characterised better.

\section{Appendices}

\section{A Numerical Implementation}

The aim of the numerical subroutine is to compute the stress given a strain increment. In our state variable 
(a)

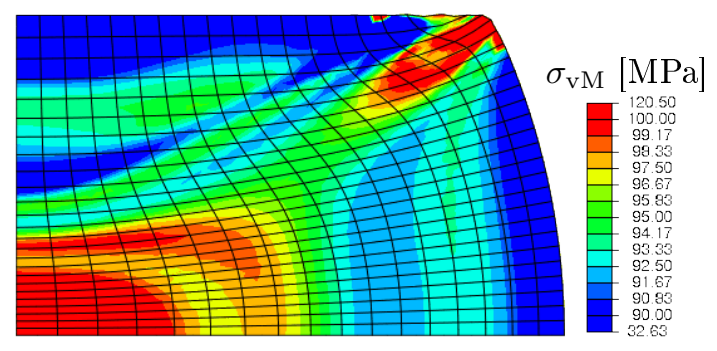

(b)

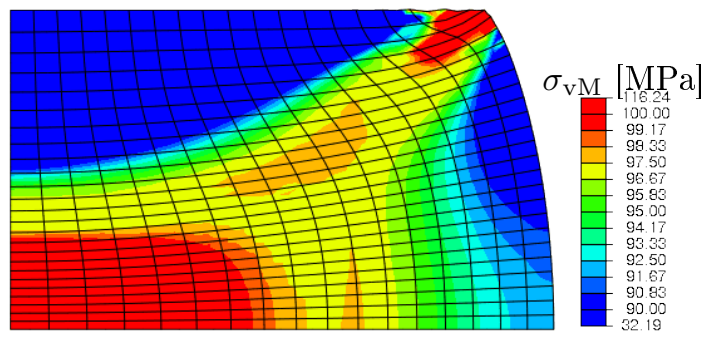

(c)

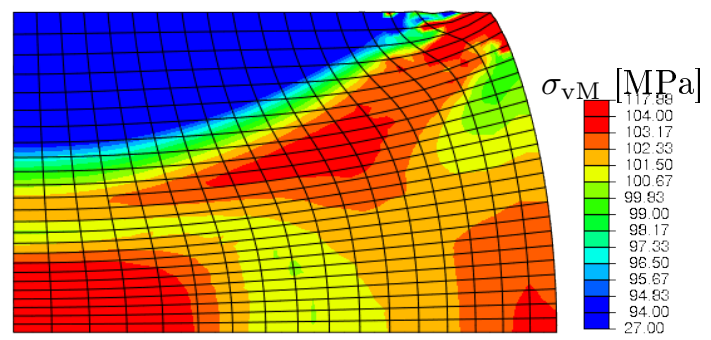

(d)

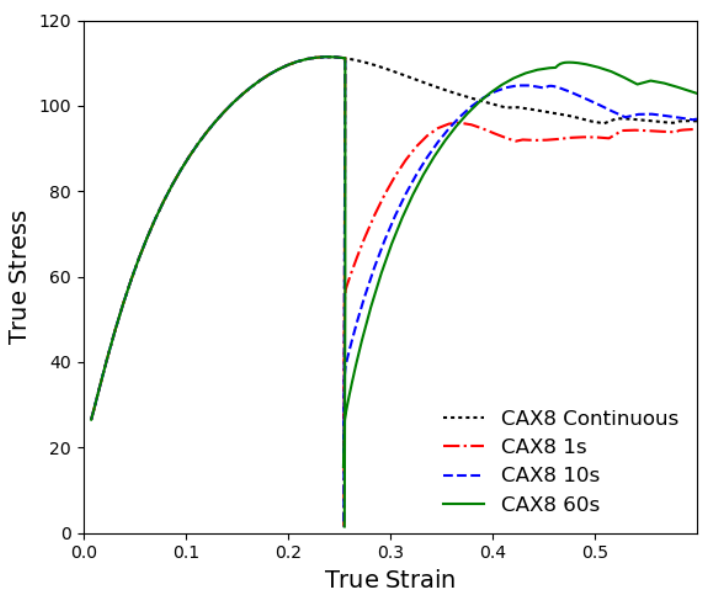

Figure 7: Von Mises stress contours using $425 \times$ full integration 8 noded axisymmetric stress elements (CAX8) after a two stage compression simulation at $541^{\circ} \mathrm{C}$. A total of $60 \%$ total reduction is applied over $0.6 \mathrm{~s}$ with a varying stress free inter-compression time at the $0.3 \mathrm{~s}$ mark. The result using an inter-compression time of (a) 1s, (b) 10s and (c) 60s illustrate the effect of static recrystallisation and history dependence using the model. The true stress - true strain curves of the continuous as well as interrupted compression simulations are given in (d). formulation the stress depends on the evolution of state variables as a function of temperature as well as incremental strain and time step. The system of differential equations that describe the evolution of the state variables need to be integrated numerically. Here, we choose the numerically stable fully implicit Backward Euler integration scheme.

Integration of the ISVs and associated stresses is implemented into an Abaqus UMAT and linked subroutines. The UMAT framework in Appendix B resolves the incremental plastic strain using calls to an FISOTROPIC subroutine added as Appendix C. In the numerical implementation, the state variables per active volume fraction in the FISOTROPIC subroutine is solved using calls to a residual subroutine RGET added as Appendix D. A flow diagram of the radial return type UMAT framework in Appendix B is given in Figure 9. This figure also illustrates the main variables of interest given as input and outputs of the subroutine. Details on the FISOTROPIC and RGET subroutines are covered later.

The model integrates the various values incrementally with previous converged ISV values stored in the STATEV array. Candidate ISV values are stored internal to the UMAT in a TEMPSTATEV array. The STATEV array is updated upon convergence using the values of the temporary ISV array. Values that are useful in an analysis apart from the ISVs needed in the recrystallisation and density ratio based evolution include the accumulated volume fraction averaged equivalent plastic strain. An ISV for the equivalent plastic strain is therefore also assigned per recrystallisation volume fraction $\alpha_{\mathrm{x}_{i}}$. This is done to keep track of the equivalent plastic strain that accumulates and is reset by each wave of recrystallisation. If a specific recrystallisation volume fraction $f_{\mathrm{x}_{i}}$ is active, the plastic strain increment $\delta \alpha$ is added to the volume growth compensated internal state variable

$$
\left.\alpha_{\mathrm{x}_{i}}\right|_{t+\delta t}=\left.\alpha_{\mathrm{x}_{i}}\right|_{t} \frac{\left.f_{\mathrm{x}_{i}}\right|_{t}}{\left.f_{\mathrm{x}_{i}}\right|_{t+\delta t}}+\delta \alpha .
$$

The values of the volume fraction averaged plastic strain and misorientation can be calculated following Eq.(30) to obtain

$$
\bar{\alpha}=\sum_{i=0}^{n_{\mathrm{x}}-1} \alpha_{\mathrm{x}_{i}}\left(f_{\mathrm{x}_{i}}-f_{\mathrm{x}_{i+1}}\right)
$$

and

$$
\bar{\lambda}=\sum_{i=0}^{n_{\mathrm{x}}-1} \lambda_{\mathrm{x}_{i}}\left(f_{\mathrm{x}_{i}}-f_{\mathrm{x}_{i+1}}\right) .
$$

The internal state variables at the end of each time increment is the volume fraction averaged plastic strain $\bar{\alpha}$, dislocation density ratio $\bar{\varrho}$ and average slip plane lattice misorientation $\bar{\lambda}$. These three averaged ISVs are followed by four ISVs per volume fraction namely the fraction specific equivalent plastic strain $\alpha_{\mathrm{x}_{i}}$, dislocation density $\varrho_{\mathrm{x}_{i}}$, misorientation $\lambda_{\mathrm{x}_{i}}$ and next volume 


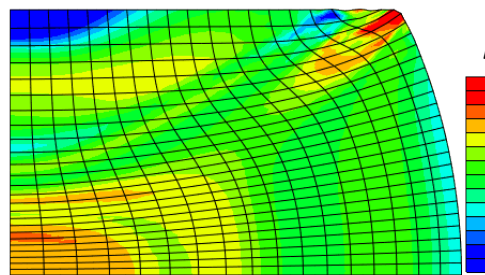

(a)

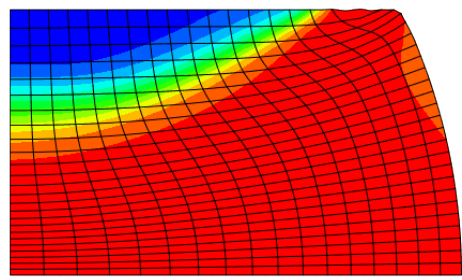

(d)

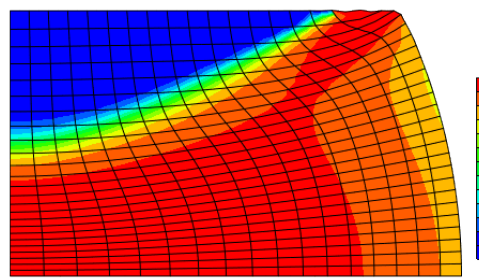

$(\mathrm{g})$

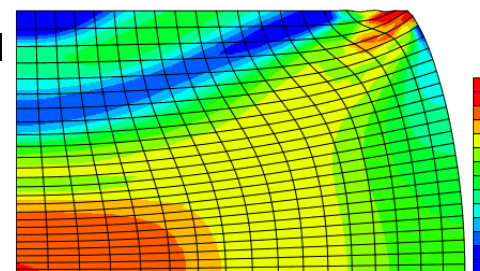

(b)
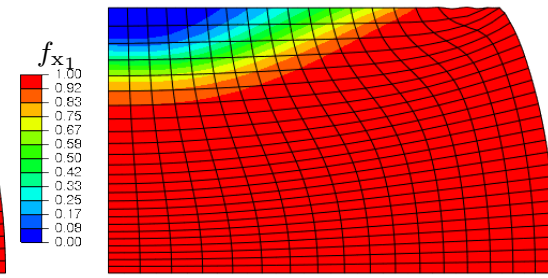

(e)
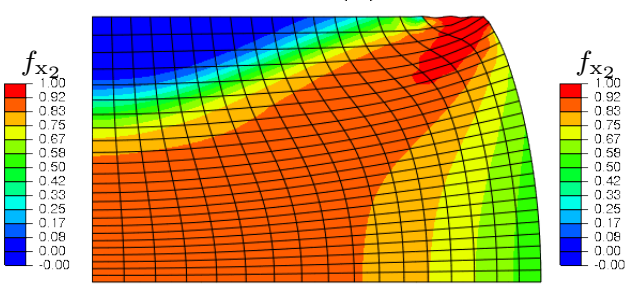

(h)

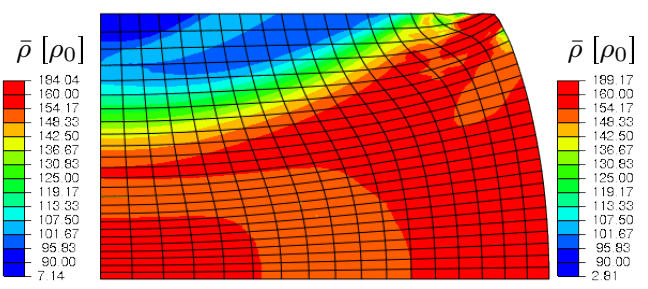

(c)
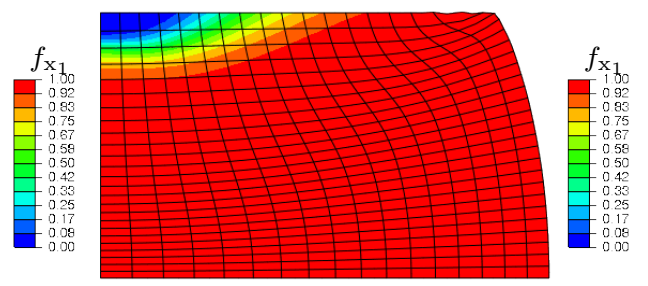

(f)

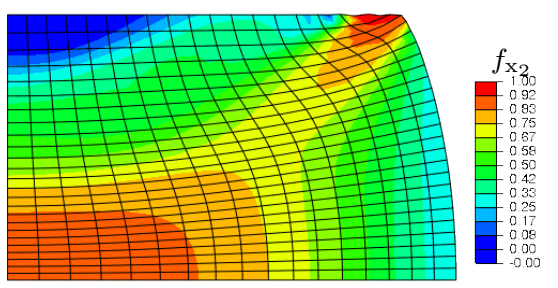

(i)

Figure 8: Contours using $425 \times \mathrm{CAX} 8$ elements after a two stage compression simulation at $541^{\circ} \mathrm{C}$. A total of $60 \%$ total reduction is applied over $0.6 \mathrm{~s}$ with a varying stress free inter-compression time at the $0.3 \mathrm{~s}$ mark. Contours of the dislocation density ratio (as a function of the original dislocation density $\rho_{0}$ ) as a result of (a) $1 \mathrm{~s}$, (b) 10s and (c) 60s inter-compression time is given. The volume fraction of material recrystallised at least once $\left(f_{\mathrm{x}_{1}}\right)$ and at least twice $\left(f_{\mathrm{x}_{2}}\right)$ are also given for the $(\mathrm{d}, \mathrm{g}) 1 \mathrm{~s},(\mathrm{e}, \mathrm{g}) 10 \mathrm{~s}$ and $(\mathrm{f}, \mathrm{i}) 60 \mathrm{~s}$ static recrystallisation cases modelled.

fraction value $f_{\mathrm{x}_{i+1}}$. All of these ISV values have to be stored in the STATEV array.

The total length of the state variable array (NSTATV) is set in an Abaqus input file with the $*$ DEPVAR card. The volume fraction averaged equivalent plastic strain, dislocation density ratio and average slip plane lattice misorientation are stored in the first three entries of the state variable array $\operatorname{STATEV}(1: 3)$. Tracking the evolution of the equivalent plastic strain, dislocation density ratio, average slip plane lattice misorientation as well as volume fraction for each recrystallisation cycle implies that four entries in the STATEV array need to be allocated per volume fraction. This means that given the maximum number of possible recrystallising volume fractions $\left(\mathrm{NRRX}=n_{\mathrm{x}}\right)$, the total length of the STATEV array (NSTATV) as given by the material definition in the Abaqus input file should be at least DEPVAR $=4 * \mathrm{NRRX}+3$ so that enough memory is allocated to the problem. The previous converged values of the volume fraction averaged quantities as well as the ISV values at the end of the previous increment are stored in the state variable array sent as input and then returned at the end of the current increment as

$\operatorname{STATEV}(1: 4 * \operatorname{NRRX}+3)=\left\{\bar{\alpha}, \bar{\varrho}, \bar{\lambda}, \alpha_{\mathrm{x}_{0}}, \varrho_{\mathrm{x}_{0}}, \lambda_{\mathrm{x}_{0}}, f_{\mathrm{x}_{1}}\right.$, $\left.\ldots, \alpha_{\mathrm{x}_{n_{\mathrm{x}}-1}}, \varrho_{\mathrm{x}_{n_{\mathrm{x}}-1}}, \lambda_{\mathrm{x}_{n_{\mathrm{x}}-1}}, f_{\mathrm{x}_{\mathrm{n}_{\mathrm{x}}}}\right\}$.
Following Brown and Bammann's approach [8], it makes no sense to evolve and update the ISV values for the original volume fraction $\varrho_{\mathrm{x}_{0}}$ and $\lambda_{\mathrm{x}_{0}}$ once it has been fully recrystallised. This happens when the first recrystallised volume fraction in the state variable array defined above approaches unity $f_{\mathrm{x}_{1}} \approx 1$. If this is the case, the state variable values associated with the first recrystallised volume fraction is shifted so that it is now associated with the new default volume fraction. If this is the case, the previous converged values in the state variable array may alternatively be considered as

$$
\begin{array}{r}
\operatorname{STATEV}(7: 4 * \operatorname{NRRX}+3)=\left\{f_{\mathrm{x}_{0}} \approx 1, \alpha_{\mathrm{x}_{0}}, \varrho_{\mathrm{x}_{0}}, \lambda_{\mathrm{x}_{0}}, f_{\mathrm{x}_{1}},\right. \\
\left.\ldots, \alpha_{\mathrm{x}_{n_{\mathrm{x}}-2}}, \varrho_{\mathrm{x}_{n_{\mathrm{x}}-2}}, \lambda_{\mathrm{x}_{n_{\mathrm{x}}-2}}, f_{\mathrm{x}_{\mathrm{n}_{\mathrm{x}}-1}}\right\} .
\end{array}
$$

To reduce the amount of allocated memory required, an ISV shift applied to the STATEV array would result in a new state variable array where

$$
\begin{array}{r}
\operatorname{STATEV}(4: 4 * \operatorname{NRRX}+3)=\{\operatorname{STATEV}(8: 4 * \operatorname{NRRX}+3) \\
0,0,0,0\}
\end{array}
$$

In this implementation, the potential state variable array shift happens before calculating the stresses associated with the current time increment and additional evolution of the ISVs.

The maximum number of volume fractions to track is 


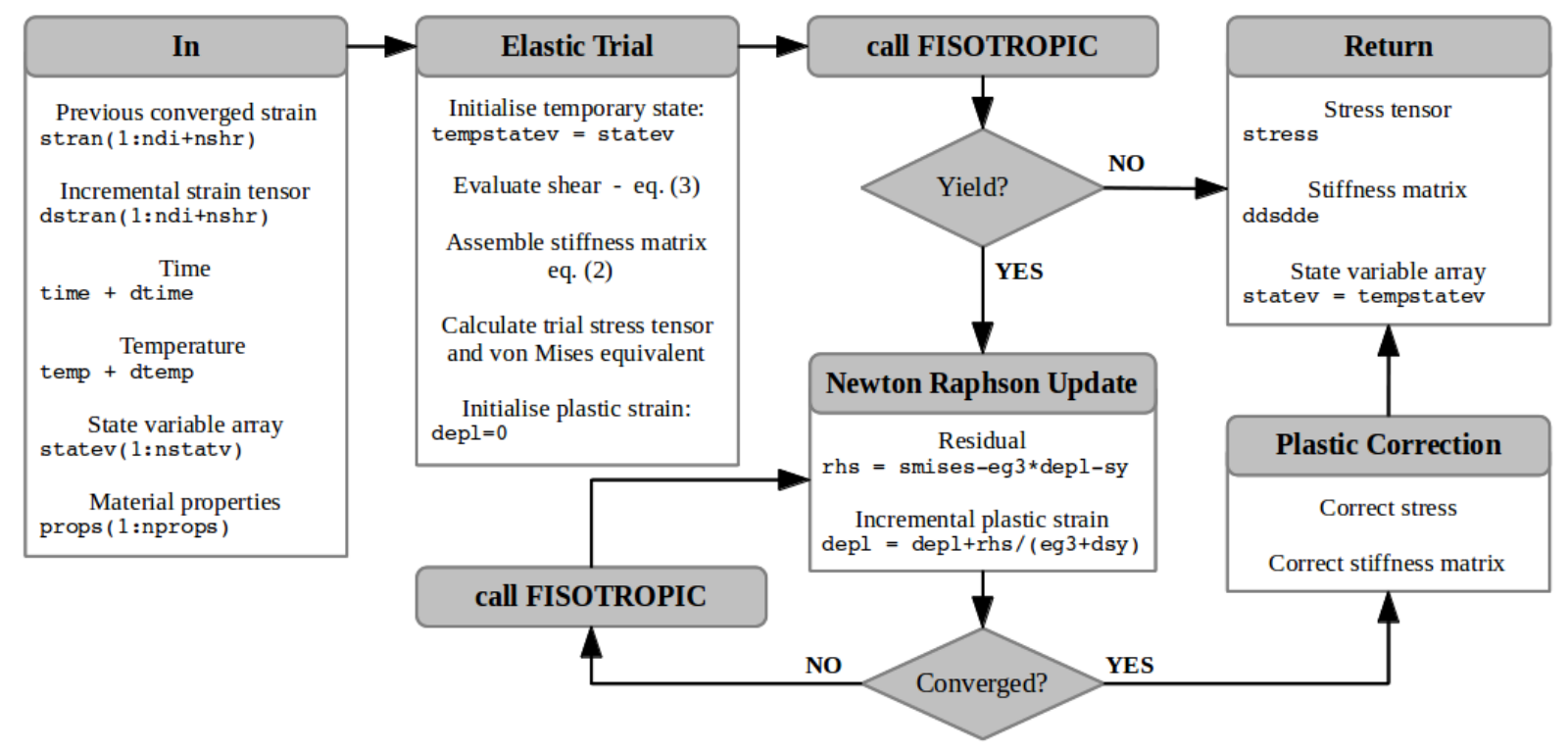

Figure 9: Diagram illustrating some of the inputs and values returned as well as the flow of calculation in the radial return type UMAT framework in Appendix B.

set with $n_{\mathrm{x}}=($ NSTATV-3) $/ 4$. The value of NSTATV is set using the $*$ DEPVAR card in the Abaqus input file, used to allocate the memory required. The volume fractions are effectively fully recrystallised and ISVs shifted once $f_{\mathrm{x}_{1}}>0.999$. ISV updates also only cycle through each of the following volume fractions as long as the conditions $f_{\mathrm{x}_{i+1}}>0.001$ and $i+1 \leq n_{\mathrm{x}}$ are met to save on computational time. This is different from the implementation by Brown and Bammann [8] in that they evolve all volume fraction state variables, even before it contributes to the overall material response.

\section{A.1 Plasticity and internal state evolu- tion}

Considering the current temperature, the shear modulus in Eq.(4) and scale function in Eq.(8) are evaluated first. To determine the yield stress from Eq.(7), the threshold stress value and therefore average dislocation density ratio at the end of the current increment are required as in Eq.(30). The numerical implementation needs to cycle through each of the recrystallised volume fractions, evolving the associated ISVs and then adding the contribution to the average dislocation density ratio. The aim within the FISOTROPIC subroutine is therefore to cycle through each active volume $f_{\mathrm{x}_{i}}>0.001$ to find converged values of $\varrho_{\mathrm{x}_{i}}, \lambda_{\mathrm{x}_{i}}$ and $f_{\mathrm{x}_{i+1}}$ by resolving calls to the RGET subroutine in Appendix D.

It is possible to solve the ISVs $\varrho_{\mathrm{x}_{i}}, \lambda_{\mathrm{x}_{i}}$ and $f_{\mathrm{x}_{i+1}}$ as a system of three equations or staggered. In the staggered approach followed here, a system of two equations is solved for $\varrho_{\mathrm{x}_{i}}$ and $\lambda_{\mathrm{x}_{i}}$ while $f_{\mathrm{x}_{i+1}}$ is solved as a function of $\varrho_{\mathrm{x}_{i}}, \lambda_{\mathrm{x}_{i}}$ and itself.
Given that the implementation cycles through the volume fractions, a single variable is used for values of the volume fractions, rates and ISVs needed within the specific cycle evaluated in an RGET call. The values are stored to the temporary state variable array before continuing to the next cycle. In the next cycle, the same variables now effectively just point to alternate entries of the state variable array. Here, we use the subscripts $\mathrm{x} c$ and $\mathrm{x} n$ to represent the variables associated with the current $c=i$ and next $n=i+1$ volume fractions.

To start the flow rule evaluation, the values associated with the default volume fraction $i=0$ are set from the known conditions $f_{\mathrm{x}_{0}}=1$ and $\dot{f}_{\mathrm{x}_{0}}=0$, meaning $f_{\mathrm{xc}}=$ 1 and $\dot{f}_{\mathrm{x} c}=0$. The volume fraction averaged quantities are also initialised with $\bar{\alpha}=0, \bar{\varrho}=0$ and $\bar{\lambda}=0$. A vector $\mathbf{x}=\left\{\varrho_{\mathbf{x}_{i}}, \lambda_{\mathbf{x}_{i}}\right\}_{t+\delta t}$ represents an estimate of the ISV values associated with the current volume fraction. The construction of the various residuals and solutions necessary to solve the ISV evolution are described next.

Considering that from Eq.(18) the misorientation fractional value $\bar{\theta} / \theta_{m}$ should be below unity, Eq.(25) is evaluated with this constraint in mind which gives

$$
C_{\mathrm{Rx} \lambda}=1-\exp \left(-C_{\mathrm{Rx} \lambda 0} \min \left(\left[x_{2}, 1\right]\right)^{r_{\mathrm{Rx} \lambda}}\right) .
$$

The recrystallised volume fraction growth associated with the next volume fraction $f_{\mathrm{x} n}$ is calculated by first setting the estimated variable value equal to the previous converged value $f_{\mathrm{x} n}=\left\{f_{\mathrm{x}_{i+1}}\right\}_{t}=\operatorname{STATEV}(4 *$ $i+7)$. The minimum value $f_{\mathrm{x} n} \geq 10^{-4}$ is introduced in the presented implementation in order to avoid a zero interface surface area when evaluating Eq.(22). This assumes that nucleation has already started. $f_{\mathrm{x} n}$ is now the current estimate of $\left\{f_{\mathrm{x}_{i+1}}\right\}_{t+\delta t}$ and the validity of this estimate is determined by evaluating the 
residual:

$$
f_{R_{\mathrm{x}}}=f_{\mathrm{x} n}-\left\{f_{\mathrm{x}_{i+1}}\right\}_{t}-\delta t \dot{f}_{\mathrm{x} n}=0
$$

where the rate of the next volume fraction is computed from Eq.(23) to give

$$
\dot{f}_{\mathrm{x} n}=x_{1} C_{\mathrm{Rx} 0} C_{\mathrm{Rx} T}(T) C_{\mathrm{Rx} \lambda}\left(x_{2}\right) g_{\mathrm{x}}\left(f_{\mathrm{x} c}, f_{\mathrm{x} n}\right) .
$$

The residual equation is solved using the NewtonRaphson method following

$$
\begin{gathered}
\left\{f_{\mathrm{x} n}\right\}^{k+1}=\left\{f_{\mathrm{x} n}\right\}^{k}-(1- \\
\left.x_{1} C_{\mathrm{Rx} 0} C_{\mathrm{Rx} T}(T) C_{\mathrm{Rx} \lambda}\left(x_{2}\right)\left\{\frac{d g_{\mathrm{x}}}{d f_{\mathrm{x} n}}\right\}^{k}\right)^{-1}\left\{f_{R_{\mathrm{x}}}\right\}^{k} .
\end{gathered}
$$

Once the next volume fraction is solved at $t+\delta t$, the residuals on the two ISV estimates can be determined using the evolution rates of Eqs.(28) and (29). This gives the following two residuals that depend on $x_{1}$ and $x_{2}$ :

$$
\begin{aligned}
& f_{R_{1}}\left(x_{1}, x_{2}\right)=x_{1}-\left.\varrho_{\mathrm{x}_{i}}\right|_{t}- \\
& \delta \alpha\left(C_{0} x_{2}^{r_{g}}+C_{1} \sqrt{x_{1}}-C_{2}(\dot{\alpha}, T) x_{1}\right)+ \\
& \delta t C_{3}(T) x_{1}^{r_{3}}+\delta t \frac{\dot{f}_{\mathrm{x} c}}{f_{\mathrm{x} c}-f_{\mathrm{x} n}\left(x_{1}, x_{2}\right)} x_{1}=0
\end{aligned}
$$

and

$$
\begin{aligned}
f_{R_{2}}\left(x_{1}, x_{2}\right)=x_{2}-\left.\lambda\right|_{t}-\delta \alpha C_{\lambda}+ & \\
\delta t \frac{\dot{f}_{\mathrm{x} c}}{f_{\mathrm{x} c}-f_{\mathrm{x} n}\left(x_{1}, x_{2}\right)} & x_{2}=0 .
\end{aligned}
$$

The ISV updates are solved by using the initial guess $\mathbf{x}=\left\{\varrho_{\mathbf{x}_{i}}, \lambda_{\mathbf{x}_{i}}\right\}_{t}=\{\operatorname{STATEV}(4 * i+5,4 * i+6)\}$, sent in to the RGET subroutine in Appendix D. The residual values and sensitivities are returned to the FISOTROPIC subroutine. The values are updated using the NewtonRaphson scheme

$$
\{\mathbf{x}\}^{k+1}=\{\mathbf{x}\}^{k}-\left[\left\{\mathbf{F}_{R}^{\prime}\right\}^{k}\right]^{-1}\left\{\mathbf{f}_{R}\right\}^{k}
$$

where the Jacobian matrix $F_{R i j}^{\prime}=\partial f_{R_{i}} / \partial x_{j}$ contains the partial derivatives of the residuals in Eqs.(42) and (43). Since we have a $2 \times 2$ system, we compute the inverse of $\mathbf{F}_{R}^{\prime}$ using the closed form expression

$$
\left[\mathbf{F}_{R}^{\prime}\right]^{-1}=\frac{1}{\operatorname{det}\left(\mathbf{F}_{R}^{\prime}\right)}\left[\begin{array}{cc}
F_{R 2,2}^{\prime} & -F_{R 1,2}^{\prime} \\
-F_{R 2,1}^{\prime} & F_{R 1,1}^{\prime}
\end{array}\right]
$$

with $\operatorname{det}\left(\mathbf{F}_{R}^{\prime}\right)=F_{R 1,1}^{\prime} F_{R 2,2}^{\prime}-F_{R 1,2}^{\prime} F_{R 2,1}^{\prime}$. The com- ponents of the Jacobian matrix are:

$$
\begin{aligned}
\frac{\partial f_{R_{1}}}{\partial x_{1}}= & 1-\delta \alpha\left(\frac{1}{2} C_{1} x_{1}^{-1 / 2}-C_{2}\right)+\delta t r_{3} C_{3} x_{1}^{r_{3}-1}+ \\
& \delta t \frac{\dot{f}_{\mathrm{x} c}}{f_{\mathrm{x} c}-f_{\mathrm{x} n}}+\delta t x_{1} \frac{\dot{f}_{\mathrm{x} c}}{\left(f_{\mathrm{x} c}-f_{\mathrm{x} n}\right)^{2}} \frac{d f_{\mathrm{x} n}}{d x_{1}} \\
\frac{\partial f_{R_{1}}}{\partial x_{2}}= & -\delta \alpha r_{g} C_{0} x_{2}^{r_{g}-1}+\delta t x_{1} \frac{\dot{f}_{\mathrm{x} c}}{\left(f_{\mathrm{x} c}-f_{\mathrm{x} n}\right)^{2}} \frac{d f_{\mathrm{x} n}}{d x_{2}} \\
\frac{\partial f_{R_{2}}}{\partial x_{1}}= & \delta t x_{2} \frac{\dot{f}_{\mathrm{x} c}}{\left(f_{\mathrm{x} c}-f_{\mathrm{x} n}\right)^{2}} \frac{d f_{\mathrm{x} n}}{d x_{1}}, \\
\frac{\partial f_{R_{2}}}{\partial x_{2}}= & 1+\delta t \frac{\dot{f}_{\mathrm{x} c}}{f_{\mathrm{x} c}-f_{\mathrm{x} n}}+\delta t x_{2} \frac{\dot{f}_{\mathrm{x} c}}{\left(f_{\mathrm{x} c}-f_{\mathrm{x} n}\right)^{2}} \frac{d f_{\mathrm{x} n}}{d x_{2}}
\end{aligned}
$$

The derivatives of the next volume fraction with respect to the current ISV estimates are determined from the residual in Eq.(39) as:

$$
\begin{aligned}
\frac{d f_{\mathrm{x} n}}{d x_{1}}= & \delta t C_{\mathrm{Rx} 0} C_{\mathrm{Rx} T}(T) C_{\mathrm{Rx} \lambda}\left(x_{2}\right) g_{\mathrm{x}}\left(f_{\mathrm{x} c}, f_{\mathrm{x} n}\right) \times \\
& \left(1-\delta t x_{1} C_{\mathrm{Rx} 0} C_{\mathrm{Rx} T}(T) C_{\mathrm{Rx} \lambda}\left(x_{2}\right) \frac{d g_{\mathrm{x}}}{d f_{\mathrm{x} n}}\right)^{-1} \\
\frac{d f_{\mathrm{x} n}}{d x_{2}}= & \delta t x_{1} C_{\mathrm{Rx} 0} C_{\mathrm{Rx} T}(T)\left(f_{\mathrm{x} c}, f_{\mathrm{x} n}\right) \frac{d C_{\mathrm{Rx} \lambda}}{d x_{2}} \times \\
& \left(1-\delta t x_{1} C_{\mathrm{Rx} 0} C_{\mathrm{Rx} T}(T) C_{\mathrm{Rx} \lambda}\left(x_{2}\right) \frac{d g_{\mathrm{x}}}{d f_{\mathrm{x} n}}\right)^{-1}
\end{aligned}
$$

and

$$
\frac{d C_{\mathrm{Rx} \lambda}}{d x_{2}}=r_{\mathrm{Rx} \lambda} C_{\mathrm{Rx} \lambda 0} x_{2}^{r_{\mathrm{Rx} \lambda}-1}\left[1-\exp \left(-C_{\mathrm{Rx} \lambda 0} x_{2}^{r_{\mathrm{Rx} \lambda}}\right)\right]
$$

if $x_{2} \leq 1$ or zero otherwise.

\section{A.2 Flow rule sensitivity}

Before possibly moving to the next volume fraction, the current volume fraction contribution to the equivalent dislocation density is needed. This is updated along with the contribution to the yield stress sensitivity required to resolve the flow rule residual and solve the equivalent plastic strain increment. The contribution to the average dislocation density ratio is calculated as in Eq.(30) by updating the value of the equivalent dislocation density ratio variable. The equivalent dislocation density ratio variable is initialised $\bar{\varrho}=0$ at the start of the calculation. For each subsequent volume fraction solved, the variable is updated following

$$
\bar{\varrho}^{k+1}=\bar{\varrho}^{k}+x_{1}\left(f_{\mathrm{x} c}-f_{\mathrm{x} n}\right)
$$

so that $\bar{\varrho}^{k}$ now represents the summation of the first $k$ volume fraction contributions $\bar{\varrho}^{k}=$ $\sum_{i=0}^{k} \varrho_{\mathrm{x}_{i}}\left(f_{\mathrm{x}_{i}}-f_{\mathrm{x}_{i+1}}\right)$.

The sensitivity of the yield stress with respect to the 
equivalent plastic strain increment in turn is given by

$$
\frac{d \sigma_{\mathrm{Y}}}{d \delta \alpha}=\frac{\sigma_{0} \sqrt{\varrho}}{\delta t} \frac{d S_{\varepsilon}}{d \dot{\alpha}}+\frac{S_{\varepsilon} \sigma_{0}}{2 \sqrt{\varrho}} \frac{d \bar{\varrho}}{d \delta \alpha}
$$

which requires $d S_{\varepsilon} / d \dot{\alpha}$ and $d \bar{\varrho} / d \delta \alpha$. The definition of the scaling factor in Eq.(8) leads to

$$
\begin{aligned}
& \frac{d S_{\varepsilon}}{d \dot{\alpha}}=\frac{T}{\dot{\alpha} p q a_{0} \mu_{r}}\left(\frac{T}{a_{0} \mu} \ln \left(\frac{\dot{\varepsilon}_{0}}{\dot{\alpha}}\right)\right)^{1 / q-1} \times \\
& {\left[1-\left(\frac{T}{a_{0} \mu} \ln \left(\frac{\dot{\varepsilon}_{0}}{\dot{\alpha}}\right)\right)^{1 / q}\right]^{1 / p-1} . }
\end{aligned}
$$

The equivalent dislocation density ratio sensitivity is computed from Eq.(30) to give

$$
\begin{array}{r}
\frac{d \varrho}{d \delta \alpha}=\sum_{i=0}^{n_{\mathrm{x}}-1}\left[\frac{d \varrho_{\mathrm{x}_{i}}}{d \delta \alpha}\left(f_{\mathrm{x}_{i}}-f_{\mathrm{x}_{i+1}}\right)+\right. \\
\left.\varrho_{\mathrm{x}_{i}}\left(\frac{d f_{\mathrm{x}_{i}}}{d \delta \alpha}-\frac{d f_{\mathrm{x}_{i+1}}}{d \delta \alpha}\right)\right] .
\end{array}
$$

From the first volume fraction condition with $f_{\mathrm{x}_{0}}=1$, it is evident that $d f_{\mathrm{x}_{0}} / d \delta \alpha=0$. The incremental contribution to the average dislocation density ratio sensitivity added at each cycle means that given the initialised variable value $\{d \bar{\varrho} / d \delta \alpha\}^{k=0}=0$, the sensitivity is updated as each cycle evaluation is completed. Following Eq.(49), this gives

$\frac{d \bar{\varrho}}{d \delta \alpha}^{k+1}={\frac{d \bar{\varrho}^{k}}{d \delta \alpha}}^{k}+\frac{d x_{1}}{d \delta \alpha}\left(f_{\mathrm{x} c}-f_{\mathrm{x} n}\right)+x_{1}\left(\frac{d f_{\mathrm{x} c}}{d \delta \alpha}-\frac{d f_{\mathrm{x} n}}{d \delta \alpha}\right)$,

where $d f_{\mathrm{xc}} / d \delta \alpha=0$ for the first volume fraction. Given the residual in Eq.(39) with $\dot{f}_{\mathrm{x} n}$ now a function of $\mathbf{x}$ according to Eq.(40) and a known value for $d f_{\mathrm{xc}} / d \delta \alpha$, the required total derivative in Eq.(53) is given by

$$
\frac{d f_{\mathrm{x} n}}{d \delta \alpha}=\frac{\partial f_{\mathrm{x} n}}{\partial x_{1}} \frac{d x_{1}}{d \delta \alpha}+\frac{\partial f_{\mathrm{x} n}}{\partial x_{2}} \frac{d x_{2}}{d \delta \alpha}+\frac{\partial f_{\mathrm{x} n}}{\partial f_{\mathrm{x} c}} \frac{d f_{\mathrm{xc}}}{d \delta \alpha} .
$$

In the first volume fraction solved the last term in Eq.(54) is zero since $d f_{\mathrm{x} c} / d \delta \alpha=0$ for a constant $f_{\mathrm{x} c}=$ 1. If the next volume fraction is active $\left(f_{\mathrm{x} n} \geq 0.001\right)$, the value of $d f_{\mathrm{x} n} / d \delta \alpha$ as calculated in Eq.(54) is transferred to the variable $d f_{\mathrm{xc}} / d \delta \alpha$ for use in the subsequent volume fraction contribution.

The sensitivities of $x_{1}$ and $x_{2}$ with respect to the equivalent plastic strain increment are approximated in the presented implementation by again using the residual equations for the evolution of the ISVs in Eqs.(42) and (43).

The updated internal state variable associated with the dislocation density ratio evolution in Eq.(42) is given by

$$
x_{1}=\left.\varrho_{\mathrm{x}_{i}}\right|_{t}+\left.\delta \theta_{x_{1}}\right|_{t+\delta t},
$$

where $\left.\delta \theta_{x_{1}}\right|_{t+\delta t}=\delta \theta_{x_{1}}\left(\delta \alpha, \delta t, \dot{\alpha}, T, x_{1}, x_{2}, f_{\mathrm{xc}}\right)$ is solved using the residual subroutine RGET. During a call to the RGET subroutine, $\left.\delta \theta_{x_{1}}\right|_{t+\delta t}$ is mainly seen as a function of the $x_{1}$ and $x_{2}$ values at the end of the increment since the other values are assumed constant during a Newton loop.

Further sensitivities are however required so that the equivalent plastic strain can be determined from within the user material framework. The total derivative of $x_{1}$ with respect to the equivalent plastic strain using Eq.(55) is determined from the chain rule

$$
\begin{array}{r}
\frac{d x_{1}}{d \delta \alpha}=\frac{\partial \delta \theta_{x_{1}}}{\partial \delta \alpha}+\frac{\partial \delta \theta_{x_{1}}}{\partial \dot{\alpha}} \frac{d \dot{\alpha}}{d \delta \alpha}+\frac{\partial \delta \theta_{x_{1}}}{\partial f_{\mathrm{xc}}} \frac{d f_{\mathrm{xc}}}{d \delta \alpha}+ \\
\frac{\partial \delta \theta_{x_{1}}}{\partial x_{1}} \frac{d x_{1}}{d \delta \alpha}+\frac{\partial \delta \theta_{x_{1}}}{\partial x_{2}} \frac{d x_{2}}{d \delta \alpha}
\end{array}
$$

The derivative of the equivalent strain rate with respect to incremental plastic strain is $d \dot{\alpha} / d \delta \alpha=1 / \delta t$. The value of $d f_{\mathrm{x} c} / d \delta \alpha$ is equal to zero in the first volume fraction due to $f_{\mathrm{x} c}=f_{\mathrm{x}_{0}}=1$. If not in the first volume fraction it is carried over from the preceding volume fraction calculation. The equivalent plastic strain increment sensitivity in this case is taken as the sensitivity of the next volume fraction as determined in the previous solution loop, i.e. $d f_{\mathrm{x} c} /\left.d \delta \alpha\right|_{f_{\mathrm{x}_{i}+1}}=d f_{\mathrm{x} n} /\left.d \delta \alpha\right|_{f_{\mathrm{x}_{i}}}$.

Rearranging Eq.(56) and noting that $\partial f_{R_{1}} / \partial x_{1} \equiv$ $\left(1-\partial \delta \theta_{x_{1}} / \partial x_{1}\right)$ and $\partial f_{R_{1}} / \partial x_{2} \equiv-\partial \delta \theta_{x_{1}} / \partial x_{2}$ in Equations (46), this implies

$$
\frac{\partial f_{R_{1}}}{\partial x_{1}} \frac{d x_{1}}{d \delta \alpha}+\frac{\partial f_{R_{1}}}{\partial x_{2}} \frac{d x_{2}}{d \delta \alpha}=\Gamma_{x_{1}} .
$$

Here $\Gamma_{x_{1}}$ contains all of the sensitivity components in Eq.(56) not associated with $x_{1}$ and $x_{2}$ :

$$
\Gamma_{x_{1}}=\frac{\partial \delta \theta_{x_{1}}}{\partial \delta \alpha}+\frac{\partial \delta \theta_{x_{1}}}{\partial \dot{\alpha}} \frac{d \dot{\alpha}}{d \delta \alpha}+\frac{\partial \delta \theta_{x_{1}}}{\partial f_{\mathrm{xc}}} \frac{d f_{\mathrm{xc}}}{d \delta \alpha} .
$$

Doing the same as in Eq.(57) for $d x_{2} / d \delta \alpha$ leads more generally to the system of equations

$$
\frac{\partial f_{R_{i}}}{\partial x_{1}} \frac{d x_{1}}{d \delta \alpha}+\frac{\partial f_{R_{i}}}{\partial x_{2}} \frac{d x_{2}}{d \delta \alpha}=\Gamma_{x_{i}}
$$

For the first residual equation $(i=1)$ the right hand side of Eq.(59) is

$$
\begin{aligned}
\Gamma_{x_{1}} \equiv & -\frac{\partial f_{R_{1}}}{\partial \delta \alpha}-\frac{\partial f_{R_{1}}}{\partial \dot{\alpha}} \frac{d \dot{\alpha}}{d \delta \alpha}-\frac{\partial f_{R_{1}}}{\partial d f_{\mathrm{x} c}} \frac{d f_{\mathrm{x} c}}{d \delta \alpha} \\
= & C_{0} x_{2}^{r_{g}}+C_{1} \sqrt{x_{1}}-C_{2} x_{1}-\dot{\alpha} \frac{d C_{2}}{d \dot{\alpha}}+ \\
& x_{1}\left[\delta t \frac{\dot{f}_{\mathrm{x} c}}{\left(f_{\mathrm{x} c}-f_{\mathrm{x} n}\right)^{2}}-\frac{1}{f_{\mathrm{x} c}-f_{\mathrm{x} n}}\right] \frac{d f_{\mathrm{x} c}}{d \delta \alpha},
\end{aligned}
$$

with

$$
\frac{d}{d \dot{\alpha}} C_{2}(\dot{\alpha}, T)=-\frac{C_{20} T}{\dot{\alpha} a_{02} \mu} \exp \left[-\frac{T}{a_{02} \mu} \ln \left(\frac{\dot{\alpha}}{\dot{\varepsilon}_{02}}\right)\right],
$$

where Eq.(14) is used for $C_{2}(\dot{\alpha}, T)$. 
The second residual has no equivalent plastic strain rate dependency. The approximate derivative of this residual equation with respect to plastic strain gives

$$
\begin{aligned}
\Gamma_{x_{2}} & \equiv-\frac{\partial f_{R_{2}}}{\partial \delta \alpha}-\frac{\partial f_{R_{2}}}{\partial d f_{\mathrm{x} c}} \frac{d f_{\mathrm{xc} c}}{d \delta \alpha} \\
& =C_{\lambda}+x_{2}\left[\delta t \frac{\dot{f}_{\mathrm{x} c}}{\left(f_{\mathrm{x} c}-f_{\mathrm{x} n}\right)^{2}}-\frac{1}{f_{\mathrm{x} c}-f_{\mathrm{x} n}}\right] \frac{d f_{\mathrm{x} c}}{d \delta \alpha} .
\end{aligned}
$$

The values of $\partial f_{R_{i}} / \partial x_{j}$ in Eq.(59) are the same components used to construct the matrix needed in Eq.(44). The relevant derivatives of the current volume fraction ISVs with respect to equivalent plastic strain are then given by

$$
\frac{d x_{1}}{d \delta \alpha}=\frac{1}{\operatorname{det}\left(\mathbf{F}_{R}^{\prime}\right)}\left(F_{R 2,2}^{\prime} \Gamma_{x_{1}}-F_{R 1,2}^{\prime} \Gamma_{x_{2}}\right)
$$

and

$$
\frac{d x_{2}}{d \delta \alpha}=\frac{1}{\operatorname{det}\left(\mathbf{F}_{R}^{\prime}\right)}\left(F_{R 1,1}^{\prime} \Gamma_{x_{2}}-F_{R 2,1}^{\prime} \Gamma_{x_{1}}\right) .
$$

The individual volume fraction sensitivities of the ISVs are then used to compute the averaged contributions of the ISVs.

The current recrystallised volume fraction compensated equivalent plastic strain using Eq.(32) is determined from $\left.\alpha_{\mathrm{x} c}\right|_{t+\delta t}=\left.\alpha_{\mathrm{x} c}\right|_{t} \times\left. f_{\mathrm{xc}}\right|_{t} /\left.f_{\mathrm{xc}}\right|_{t+\delta t}+\delta \alpha$. The converged value of the plastic strain for the previous increment is $\left.\alpha_{\mathrm{x} c}\right|_{t}=\operatorname{STATEV}(4 * i+4)$ while that for the current volume fraction is $\left.f_{\mathrm{x} c}\right|_{t}=\operatorname{STATEV}(4 * \mathrm{i}$ $+7)$. Starting with $\bar{\alpha}^{k=0}=0$ and $\bar{\lambda}^{k=0}=0$, the volume averaged equivalent plastic strain and average slip plane lattice misorientation are updated in the same way as the equivalent dislocation density in Eq.(49):

$$
\begin{aligned}
& \bar{\alpha}^{k+1}=\bar{\alpha}^{k}+\alpha_{\mathrm{xc}}\left(f_{\mathrm{x} c}-f_{\mathrm{x} n}\right), \\
& \bar{\lambda}^{k+1}=\bar{\lambda}^{k}+x_{2}\left(f_{\mathrm{x} c}-f_{\mathrm{x} n}\right) .
\end{aligned}
$$

Once all of the current volume fraction contributions are accounted for, the current volume fraction values are stored in the associated temporary state variable locations

$$
\begin{aligned}
& \operatorname{TEMPSTATEV}(4 * i+4)=\alpha_{\mathrm{xc}} \\
& \operatorname{TEMPSTATEV}(4 * i+5)=x_{1} \\
& \operatorname{TEMPSTATEV}(4 * i+6)=x_{2} \\
& \operatorname{TEMPSTATEV}(4 * i+7)=f_{\mathrm{x} n} .
\end{aligned}
$$

A check is performed to decide whether additional volume fractions need to be considered by evaluating $i \leq$ NRRX -1 . In the implementation here, different from the implementation by Brown and Bammann [8], the condition $f_{\mathrm{x} n} \geq 0.001$ is also evaluated since it makes no sense to spend computational power to evaluate the next volume fraction if it does not contribute to the global response. If both these conditions are met, the variables are updated for the next volume fraction evaluation with $f_{\mathrm{x} c}=f_{\mathrm{x} n}, \dot{f}_{\mathrm{x} c}=\dot{f}_{\mathrm{x} n}$, $d f_{\mathrm{x} c} / d \delta \alpha=d f_{\mathrm{x} n} / d \delta \alpha$ and $i=i+1$. Setting the initial guess $\mathbf{x}=\{\operatorname{STATEV}(4 * i+5,4 * i+6)\}$ subject to $x_{1} \geq 1$ and $x_{2} \geq 10^{-4}$, the evaluation of the next volume fraction is considered by again starting at Eq.(38).

A flow chart of the current subsection regarding the evaluation of the ISV evolution, resulting yield stress and sensitivity as implemented in Appendix C and D are displayed in Figure 10.

\section{B : UMAT subroutine}

subroutine umat (stress, statev, ddsdde, sse, \& spd, scd, rpl, ddsddt, drplde, drpldt, stran, \& dstran, time, dtime, temp, dtemp, predef, \& dpred, cmname, ndi, nshr, ntens, nstatv, \& props, nprops, coords, drot, pnewdt, celent, \& df0, df1, noel, npt, layer, kspt, kstep, kinc)

implicit $\mathrm{real} * 8(\mathrm{a}-\mathrm{h}, \mathrm{o}-\mathrm{z})$

character $* 8$ cmname

dimension stress(ntens), statev(nstatv),

\& ddsdde(ntens, ntens), ddsddt (ntens),

\& drplde(ntens), stran(ntens), dstran(ntens),

\& predef (1), dpred(1), props (nprops), coords (3),

$\& \operatorname{drot}(3,3), \operatorname{df} 0(3,3), \operatorname{df} 1(3,3)$, flow $(6)$,

\& tempstatv(nstatv)

C

parameter $($ zero $=0 . \mathrm{d} 0$, one $=1 . \mathrm{d} 0, \mathrm{two}=2 . \mathrm{d} 0$, \& three $=3 . \mathrm{d} 0, \mathrm{six}=6 . \mathrm{d} 0$, enumax $=.4999 \mathrm{~d} 0$,

\& newton $=10$, toler $=1.0 \mathrm{~d}-6$ )

c assign temporary state variables

do $\mathrm{k} 1=1$, nstatv

tempstat $\mathrm{v}(\mathrm{k} 1)=$ statev $(\mathrm{k} 1)$

enddo

C

c elastic properties:

emu $0=\operatorname{props}(1)$

ed $0=\operatorname{props}(2)$

et $0=\operatorname{props}(3)$

enu $=\operatorname{props}(4)$

c temperature dependent shear model

if (temp.gt .et0) then

emu $=$ emu $0-\operatorname{ed} 0 /(\operatorname{dexp}($ et $0 /$ temp $)-$ one $)$

else

emu $=$ emu 0

endif

eg $2=$ two*emu

eg $3=$ three $*$ emu

emod $=\operatorname{eg} 2 *($ one + enu $)$

ebulk3=emod / (one-two*enu)

elam $=($ ebulk3-eg2) /three

$c$

c elastic stiffness

do $k 1=1$, ndi

do $\mathrm{k} 2=1$, ndi

$\operatorname{ddsdde}(\mathrm{k} 2, \mathrm{k} 1)=\mathrm{elam}$ 


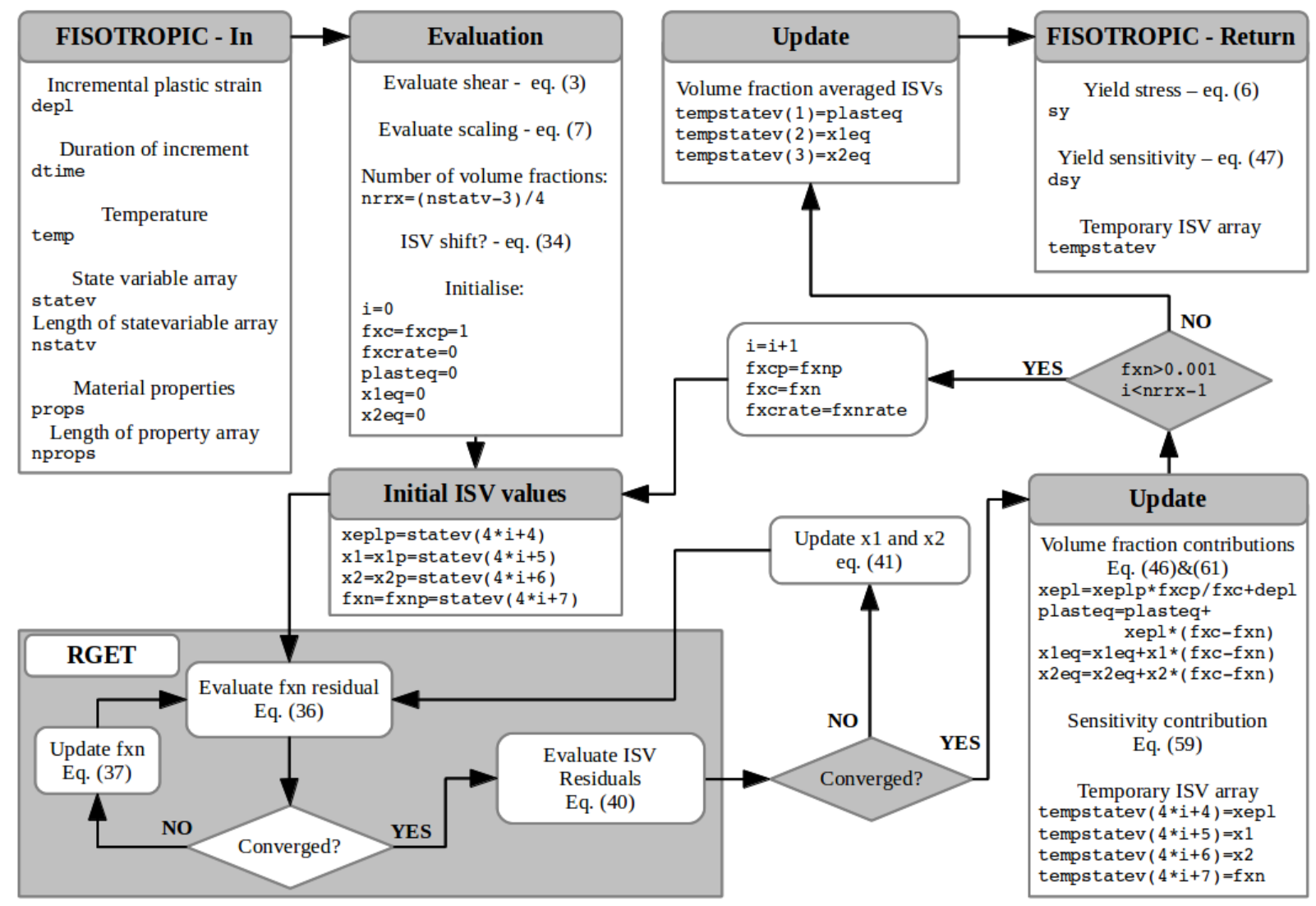

Figure 10: Flow chart sequence to solve a yield stress evaluation using the subroutines in Appendix C and D.

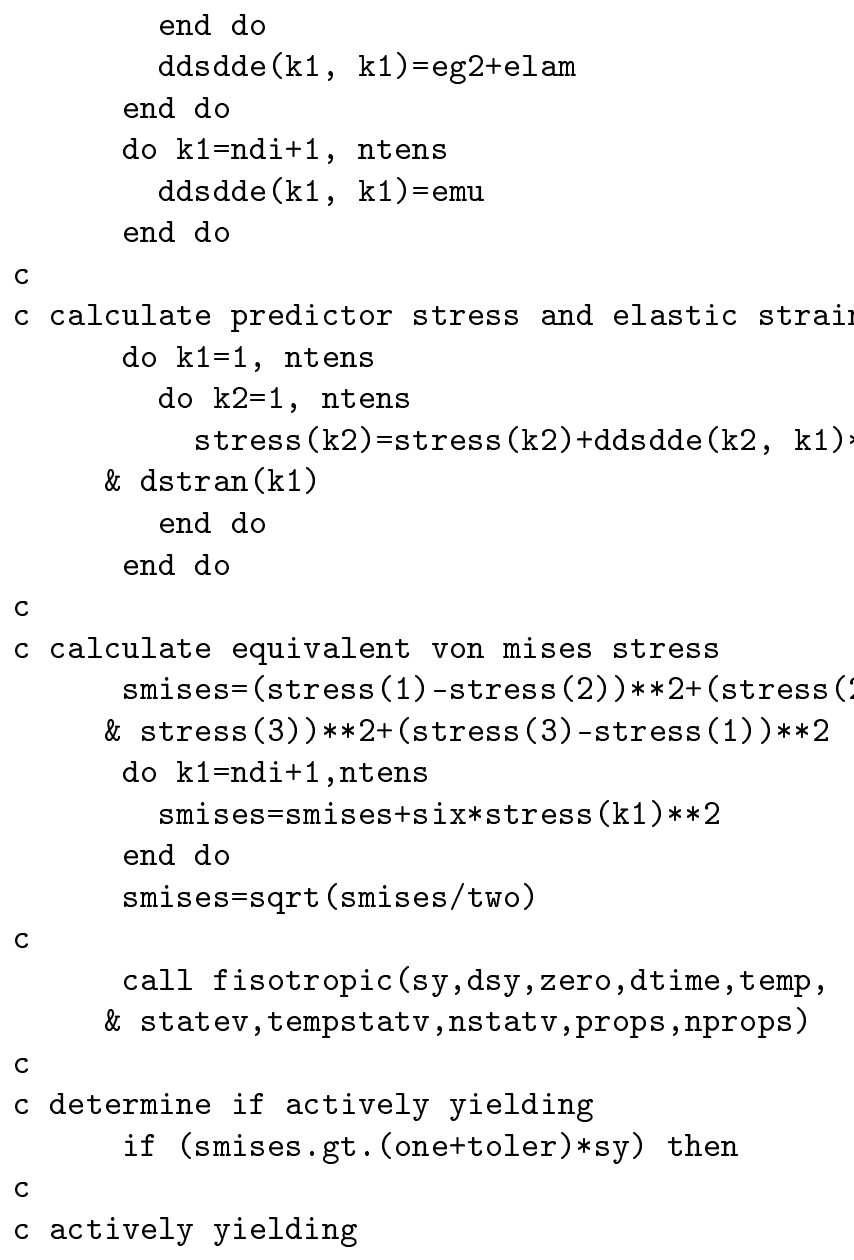

c separate the hydrostatic from the deviatoric c calculate the flow direction shydro $=($ stress (1) + stress (2) +stress (3)) / three do $\mathrm{k} 1=1$, ndi

flow $(k 1)=($ stress $(k 1)-$ shydro $) /$ smises end do do $\mathrm{k} 1=$ ndi+1, ntens

flow $(k 1)=$ stress $(k 1) /$ smises end do

C

c solve for equivalent von mises stress

$c$ and equivalent plastic strain increment using

$c$ newton iteration deqpl=zero do kewton=1, newton rhs $=$ smises - eg $3 *$ deqpl - sy deqpl=deqpl+rhs $/($ eg3+dsy $)$

call fisotropic(sy, dsy, deqpl, dtime, temp, \& statev, tempstatv, nstatv, props, nprops) if (abs (rhs).lt.toler) goto 10 end do

C

c write warning message to .msg file write $(7,2)$ newt on

2 format (//,30x,'***warning - plasticity ', \& 'algorithm did not converge after ',i3, \& ' iterations')

10 continue

c

c update stress, elastic and plastic strains and 
c equivalent plastic strain

do $\mathrm{k} 1=1$, ndi

stress $(\mathrm{k} 1)=$ flow $(\mathrm{k} 1) *$ sy+shydro

end do

do $\mathrm{k} 1=$ ndi+1,ntens

stress $(k 1)=$ flow $(k 1) * s y$

end do

C

c formulate the jacobian (material tangent)

c first calculate effective moduli

ef $f g=e m u * s y / s m i s e s$

effg2=two*effg

effg $3=$ three/two*effg2

efflam $=($ ebulk3-effg2) $/$ three

effhrd=eg $3 * d s y /($ eg $3+d s y)-e f f g 3$

do $\mathrm{k} 1=1$, ndi

do $\mathrm{k} 2=1$, ndi

$\operatorname{ddsdde}(\mathrm{k} 2, \mathrm{k} 1)=\mathrm{efflam}$

end do

ddsdde $(\mathrm{k} 1, \mathrm{k} 1)=\mathrm{effg} 2+\mathrm{efflam}$

end do

do $\mathrm{k} 1=$ ndi+1, ntens

$\operatorname{ddsdde}(\mathrm{k} 1, \mathrm{k} 1)=\mathrm{effg}$

end do

do $\mathrm{k} 1=1$, ntens

do $\mathrm{k} 2=1$, ntens

$\operatorname{ddsdde}(\mathrm{k} 2, \mathrm{k} 1)=\operatorname{ddsdde}(\mathrm{k} 2, \mathrm{k} 1)+$

\& effhrd*flow $(\mathrm{k} 2) *$ flow $(\mathrm{k} 1)$

end do

end do

endif

c

c update state variable array

do $\mathrm{k} 1=1$, nstatv

statev $(\mathrm{k} 1)=$ tempstatv $(\mathrm{k} 1)$

enddo

c

return

end

C

C : FISOTROPIC Subroutine

subroutine fisotropic (sy,dsy,depl,dtime,temp,

\& statev, tempstatev,nstatv,props,nprops)

c

implicit real*8(a-h,o-z)

logical checkrx

dimension props (nprops), statev(nstatv),

\& tempstatev(nstatv) $x i(2), x j(2), r(2), d r d x(2,2), d x 1$ eqde $=$ zero

\& fxinfo(3), reps (2), $d x d r(2,2), f \operatorname{xnvec}(5), x j u p d(2) p l a s t i c=$ zero

parameter $($ zero $=0 . d 0$, half $=0.5 \mathrm{~d} 0$, one $=1 . \mathrm{d} 0$, $\&$ two $=2 . d 0$, toler $=1 . d-4, x 10=$ one, $x 20=1 . d-10$,

\& $f \times n 0=1 . d-4$, ratelim $=1 . d-8$ )

$\mathrm{C}$

C elastic properties:

emu0 $=\operatorname{props}(1)$ c

ed $0=\operatorname{props}(2)$

et $0=\operatorname{props}(3)$

enu $=\operatorname{props}(4)$

c reference stress values

siga $=$ props $(5)$

sig0 $=\operatorname{props}(6)$

c scaling function

$\mathrm{a} 0 \mathrm{e}=\operatorname{props}(7)$

rate $0=\operatorname{props}(8)$

qe $=\operatorname{props}(9)$

pe $=\operatorname{props}(10)$

rate $=\operatorname{depl} /$ dtime

if (rate.lt.ratelim) then

rate $=$ ratelim

endif

if (temp.gt . et 0$)$ then

emu $=$ emu $0-\operatorname{ed} 0 /(\operatorname{dexp}($ et $0 /$ temp $)-$ one $)$

sfe 0 temp $/(\mathrm{a} 0$ e*emu $)$

else

$\mathrm{emu}=\mathrm{emu} 0$

sfe0 $=$ one $/ \mathrm{aOe}$

endif

emusf $=\mathrm{emu} / \mathrm{emu} 0$

C

$c$ isv shift

sfel $=\operatorname{dlog}($ rate $0 /$ rate $) * s f e 0$

sfe $=\operatorname{dabs}($ one-sfel** (one/qe) $) * *(o n e / p e)$

nrrx $=$ (nstatv-3) $/ 4$

if (statev (7).gt . (0.999d0)) then

ixvf $=1$

do while(ixvf.lt.nrrx)

lstskip $=4 *(i x v f-1)+3$

$i x v f=i x v f+1$

statev $($ lstskip +1$)=$ statev $($ lstskip +5$)$

statev $($ lstskip +2$)=$ statev $($ lstskip +6$)$

statev $($ lstskip +3$)=$ statev $($ lstskip +7$)$

statev $($ lstskip +4$)=$ statev $($ lstskip +8$)$

enddo

statev $($ lstskip +5$)=$ zero

statev (1stskip+6)=zero

statev $($ lstskip +7$)=$ zero

statev $($ lstskip +8$)=$ zero

endif

$\mathrm{fxc}=$ one

$\mathrm{fxcp}=$ one

$\mathrm{fxcr}=$ zero

dfxcde $=$ zero

$\mathrm{x} 1 \mathrm{eq}=$ zero

$\mathrm{x} 2 \mathrm{eq}=$ zero

$i x v f=1$

checkrx $=$.true.

do while((ixvf.lt.nrrx). and. (checkrx))

lstskip $=4 *(i x v f-1)+3$

xeplp = statev (lstskip+1)

$\mathrm{x} 1 \mathrm{p}=\max ($ statev $($ lstskip +2$), \mathrm{x} 10)$ 
$\mathrm{x} 2 \mathrm{p}=\max ($ statev $($ lstskip +3$), \mathrm{x} 20)$

$\mathrm{fxnp}=\max ($ statev $($ lstskip +4$), \mathrm{fxn} 0)$

$\mathrm{xi}=(/ \mathrm{x} 1 \mathrm{p}, \mathrm{x} 2 \mathrm{p} /)$

$x j=(/ x 1 p, x 2 p /)$

fxinfo $=(/ f x c, f x c r, f x n p /)$

call rget ( $r, d r d x, f x n v e c, r e p s, x j, x i, f x i n f o$,

\& depl,dtime,temp,props,nprops)

$\mathrm{fx}=\operatorname{dsqrt}(r(1) * r(1)+r(2) * r(2))$

$f \mathrm{xd}=\operatorname{drdx}(1,1) * \operatorname{drdx}(2,2)-\operatorname{drdx}(2,1) * \operatorname{drdx}(1,2)$

icount $=0$

newtmax $=15$

if ( $x i(1)$.eq. (one)) then

newtmax $=50$

endif

do while((icount.lt.newtmax).and.

\& (dabs ( $f x)$.ge.toler))

icount $=$ icount +1

if (dabs ( $f x d) \cdot g t \cdot z e r o)$ then

$d x d r=\operatorname{reshape}((/ \operatorname{drdx}(2,2),-d r d x(2,1), \quad c$

\& $\quad-\operatorname{drdx}(1,2), d r d x(1,1) /),(/ 2,2 /)) / f x d$

$\mathrm{xjupd}=\operatorname{reshape}(\operatorname{matmul}(\mathrm{dxdr}, \operatorname{reshape}(\mathrm{r},(/ 2$,

$\& 1 /())),(/ 2 /))$

$x j=x j-x j u p d$

$x j=(/ \max (\operatorname{dabs}(x j(1)), x 10), \max (\operatorname{dabs}(x j(2))$,

$\& \times 20) /$ )

fxinfo $=(/ \mathrm{fxc}, \mathrm{fxcr}, \mathrm{fxnp} /)$

call rget $(r, d r d x, f x n v e c, r e p s, x j, x i, f x i n f o$,

\& depl,dtime, temp,props,nprops)

$\mathrm{fx}=\operatorname{dsqrt}(r(1) * r(1)+r(2) * r(2))$

$f x d=\operatorname{drdx}(1,1) * d r d x(2,2)-\operatorname{drdx}(2,1) * d r d x(1, \beta)$ ar

else

$\mathrm{xj}=(/ \mathrm{x} 1 \mathrm{p}, \mathrm{x} 2 \mathrm{p} /)$

$\mathrm{fx}=$ zero

endif

enddo

$\mathrm{x} 1=\max (\mathrm{xj}(1), \mathrm{x} 10)$

$\mathrm{x} 2=\max (\mathrm{xj}(2), \mathrm{x} 20)$

$f \times n=\min (\operatorname{dabs}(f \operatorname{xnvec}(1))$, one $)$

C

c add $\mathrm{fxc}$ contribution to Gamma

$\mathrm{rxO}=$ one $/(\mathrm{fxc}-\mathrm{fxn})$

$\mathrm{dxdfxc0}=\mathrm{dfxcde} *(\mathrm{dtime} * \mathrm{fxcr} * \mathrm{rx} 0 * \mathrm{rx} 0-\mathrm{rx} 0)$

$\mathrm{dx} 1 \mathrm{dfxc}=\mathrm{dxdfxc} 0 * \mathrm{x} 1$

$\mathrm{dx} 2 \mathrm{dfxc}=\mathrm{dxdfxc} 0 * \mathrm{x} 2$

reps $=r e p s+(/ d x 1 d f x c, d x 2 d f x c /)$

C

if ( $\mathrm{fxn} . \mathrm{le} \cdot(1 \cdot \mathrm{d}-3))$ then

checkrx $=$.false.

endif

$\mathrm{fxnr}=\mathrm{fxnvec}(2)$

$\mathrm{df} \operatorname{xndx} 1=\mathrm{fxnvec}(3)$

$\mathrm{df} x \mathrm{xndx} 2=$ fxnvec $(4)$

$\mathrm{dfxndfxc}=\mathrm{fxnvec}(5)$

$\mathrm{xepl}=\mathrm{xepl} \mathbf{p} * \mathrm{fxcp} / \mathrm{fxc}+\mathrm{depl}$

C

tempstatev(1stskip+1) $=$ xepl

tempstatev(lstskip +2$)=x 1$

tempstatev(lstskip +3$)=x 2$

tempstatev(lstskip+4) $=f \times n$
C

$\mathrm{x} 1$ eq $=\mathrm{x} 1 \mathrm{eq}+\mathrm{x} 1 *(\mathrm{fxc}-\mathrm{fxn})$

$\mathrm{x} 2 \mathrm{eq}=\mathrm{x} 2 \mathrm{eq}+\mathrm{x} 2 *(\mathrm{fxc}-\mathrm{fxn})$

plastic $=$ plastic $+\operatorname{xepl} *(f x c-f x n)$

if (dabs ( $f x d)$.gt .0) then

$\mathrm{dx} 1 \mathrm{de}=\mathrm{dxdr}(1,1) * \operatorname{reps}(1)+\mathrm{dxdr}(1,2) * \operatorname{reps}(2)$

$\mathrm{dx} 2 \mathrm{de}=\mathrm{dxdr}(2,1) * \operatorname{reps}(1)+\mathrm{dxdr}(2,2) * \operatorname{reps}(2)$

$\mathrm{df} x$ nde $=\mathrm{dfxndx} 1 * \mathrm{dx} 1 \mathrm{de}+\mathrm{df} \mathrm{xndx} 2 * \mathrm{dx} 2 \mathrm{de}+$

\& dfxndfxc*dfxcde

$\mathrm{dx} 1$ eqde $=\mathrm{dx} 1$ eqde $+\mathrm{dx} 1 \mathrm{de} *(\mathrm{fxc}-\mathrm{fxn})+$

\& $\quad \mathrm{x} 1 *(\mathrm{dfxcde}-\mathrm{dfxnde})$

$\mathrm{dfxcde}=\mathrm{dfxnde}$

$f x c=f x n$

$\mathrm{fxcp}=\mathrm{fxnp}$

$\mathrm{fxcr}=\mathrm{fxnr}$

endif

endif

$i x v f=i x v f+1$

end do

tempstatev(1) = plastic

tempstatev $(2)=\mathrm{x} 1$ eq

tempstatev $(3)=\mathrm{x} 2 \mathrm{eq}$

$\operatorname{sqx} 1=\operatorname{dsqrt}(\mathrm{x} 1 \mathrm{eq})$

$\sec =\operatorname{sig} 0 * \operatorname{sq} x 1$

sy $=$ siga + emusf*sfe*sec

derivatives

$d(\mathrm{sec}) / \mathrm{d}(\mathrm{epl})$

dsecdepl = half $*$ sig $0 * d x 1$ eqde/sqx 1

c $\quad d($ sfe $) / d(e p l)$

dsfedepl $=$ (sfe0* (one-sfel** (one/qe)

\& )**(one/pe-one)*sfel**(one/qe-one)/

$\&($ pe*qe*rate $)) /$ dtime

$c \quad$ total

dsy $=$ emusf*(sfe*dsecdepl+dsfedepl $*$ sec $)$

return

end

\section{D : RGET Subroutine}

subroutine rget ( $r, d r d x j, f x n v e c, r e p s, x j, x i$, \& fxinfo, depl,dtime,temp,props,nprops)

C implicit real*8(a-h,o-z)

dimension props (nprops) $, r(2), \operatorname{drdxj}(2,2)$,

\& fxinfo(3), reps (2), xj(2), xi(2), fxnvec(5)

C

parameter (zero $=0 . \mathrm{d} 0$, half $=0.5 \mathrm{~d} 0$, one $=1 . \mathrm{d} 0$, \& two $=2 . d 0$, toler $=1 . d-10$, ratelim $=1 . d-8$ )

c elastic properties:

emu $0=\operatorname{props}(1)$

ed0 $=\operatorname{props}(2)$

et $0=\operatorname{props}(3)$ 


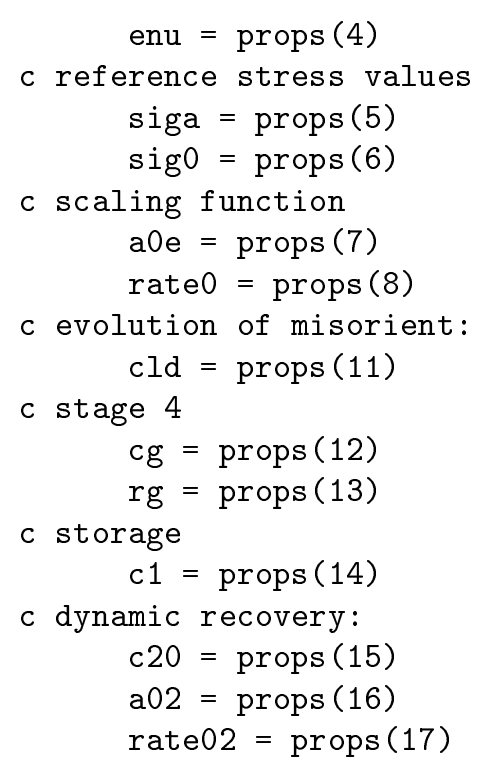

c thermal recovery c30 $=\operatorname{props}(18)$ r3 $=\operatorname{props}(19)$ $\mathrm{a} 03=\operatorname{props}(20)$

c recrystallisation $\mathrm{cx} 0=\operatorname{props}(21)$ $\mathrm{a} 0 \mathrm{x}=\operatorname{props}(22)$ $\mathrm{cxl}=\operatorname{props}(23)$ $\mathrm{rxl}=\operatorname{props}(24)$ rxa $=$ props (25) $\mathrm{rxb}=\operatorname{props}(26)$ cxc $=\operatorname{props}(27)$

C

c get info from previous values:

c contained in fxinfo:

$f x c=f x i n f o(1)$

$\mathrm{fxcr}=\operatorname{dabs}(\mathrm{fxinfo}(2))$

$f x n p=f x i n f o(3)$

c

rate $=\mathrm{depl} / \mathrm{dtime}$

if (rate.lt.ratelim) then

rate $=$ ratelim

endif

C

if (temp.gt .et0) then

emu $=$ emu $0-\operatorname{ed} 0 /(\operatorname{dexp}($ et0/temp $)-$ one $)$

$\mathrm{r} 2 \mathrm{~m}=-\mathrm{temp} /(\mathrm{emu} * \mathrm{a} 02)$

$\mathrm{r} 3 \mathrm{c}=\mathrm{c} 30 * \operatorname{dexp}(-\mathrm{a} 03 / \mathrm{temp}) * \operatorname{dtime}$

$\mathrm{r} 5 \mathrm{c} 0=\mathrm{cx} 0 * \operatorname{dexp}(-\mathrm{a} 0 \mathrm{x} /$ temp $) *$ emu $*$ dtime

else

c use constants:

$\mathrm{emu}=\mathrm{emu} 0$

$\mathrm{r} 2 \mathrm{~m}=-\mathrm{a} 02$

$\mathrm{r} 3 \mathrm{c}=\mathrm{c} 30 *$ dtime

$\mathrm{r} 5 \mathrm{c0}=\mathrm{cx} 0 * \mathrm{emu} * \mathrm{dtime}$ endif

c isv's at previous convergence and current guess:

$\mathrm{x} 1 \mathrm{p}=\max (\mathrm{xi}(1)$, one $)$

$\mathrm{x} 2 \mathrm{p}=\max (\mathrm{xi}(2)$, zero $)$

$\mathrm{x} 1=\max (\mathrm{xj}(1)$, one $)$

$\mathrm{x} 2=\max (\mathrm{xj}(2)$, zero $)$

C

c growth of next recrystallised volume: cldbar $=\min (\mathrm{x} 2$, one $)$

$\mathrm{r} 5 \mathrm{c} 1=($ one $-\operatorname{dexp}(-\mathrm{cxl} *(\mathrm{cldbar}) * * \mathrm{rxl}))$

$r 5 c=r 5 c 0 * r 5 c 1 *(x 1)$

c interfacial area

$\mathrm{fxn}=\mathrm{fxnp}$

$\mathrm{rxg}=\mathrm{fxc} *((\mathrm{fxn} / \mathrm{fxc}) * * \mathrm{rxa}) *(($ one $-\mathrm{fxn} / \mathrm{fxc}) * *$

\& $\mathrm{rxb}) *($ one $+\mathrm{cxc} *($ one-fxc) $)$

$\mathrm{drxg}=\operatorname{rxa} *((\mathrm{fxn} / \mathrm{fxc}) * *(\mathrm{rxa}-\mathrm{one})) *$

$\&(($ one $-\mathrm{fxn} / \mathrm{fxc}) * * r \mathrm{xb}) *($ one $+\mathrm{cxc} *($ one $-\mathrm{fxc}))$ -

$\& \mathrm{rxb} *((\mathrm{fxn} / \mathrm{fxc}) * * \mathrm{rxa}) *(($ one $-\mathrm{fxn} / \mathrm{fxc}) * *(\mathrm{rxb}-$

\& one $) *($ one+cxc* (one-fxc) $)$

$\mathrm{fxnr}=\mathrm{dabs}(r 5 \mathrm{c} * r \mathrm{xg})$

$f f x n=f x n-f x n p-f x n r$

c resolve residual

icount $=0$

do while((icount.lt.15). and. (dabs (ffxn).gt.

\& toler))

icount $=$ icount +1

$\mathrm{dffxn}=$ one - half $* \mathrm{r} 5 \mathrm{c} * \mathrm{drxg}$

if (dabs (dffxn).lt.toler) then

dffxn $=$ toler

endif

$f x n=\min (d a b s(f x n-f f x n / d f f x n), f x c-t o l e r)$

$\mathrm{rxg}=\mathrm{fxc} *((\mathrm{fxn} / \mathrm{fxc}) * * \mathrm{rxa}) *(($ one $-\mathrm{fxn} / \mathrm{fxc})$

$\& * * r x b) *($ one $+c x c *(o n e-f x c))$

$\mathrm{drxg}=\operatorname{rxa} *((\mathrm{fxn} / \mathrm{fxc}) * *(\mathrm{rxa}-\mathrm{one})) *$

$\&(($ one $-\mathrm{fxn} / \mathrm{fxc}) * * \mathrm{rxb}) *($ one $+\mathrm{cxc} *($ one $-\mathrm{fxc}))-$

\& $\mathrm{rxb} *((\mathrm{fxn} / \mathrm{fxc}) * * \mathrm{rxa}) *(($ one $-\mathrm{fxn} / \mathrm{fxc}) * *$

$\&(r x b-o n e)) *(o n e+c x c *(o n e-f x c))$

$\mathrm{fxnr}=\operatorname{dabs}(r 5 \mathrm{c} * r \times g)$

$f f x n=f x n-f x n p-f x n r$

end do

c partial : change of $f x n$ with respect to $x 1$ and $x 2$ :

c partial gradients $d(f x n) / d(x 1)$

ddfxdr $=$ (one-r5c*drxg)

if (dabs (ddfxdr).1t.toler) then

ddf $x d r=$ toler

endif

df $x$ ndx $1=(r 5 c 0 * r 5 c 1 * r x g) / d d f x d r$

dmdx2 = zero

if ( $\mathrm{x} 2.1 \mathrm{t}$. one) then

$\mathrm{dmdx} 2=$ one

endif

$\mathrm{dr} 5 \mathrm{c} 1 \mathrm{dm}=\mathrm{rxl} * \mathrm{cxl} * \mathrm{dexp}(-\mathrm{cxl} *(\mathrm{cldbar}) * * \mathrm{rxl}) *$

\& (cldbar)**(rxl-one)

$\mathrm{dfxndm}=(\mathrm{r} 5 \mathrm{c} 0 * \mathrm{dr} 5 \mathrm{c} 1 \mathrm{dm} * \mathrm{x} 1 * \mathrm{rxg}) / \mathrm{ddf} \mathrm{xdr}$

c partial gradients $d(f x n) / d(x 2)$

$\mathrm{df} x \mathrm{xnd} 2=\mathrm{dfxndm} * \mathrm{dmdx} 2$

c partial $d(f \times n) / d(f x c)$

$\operatorname{drxgdfxc}=((\mathrm{fxn} / \mathrm{fxc}) * * \mathrm{rxa}) *(($ one $-\mathrm{fxn} / \mathrm{fxc}) * *$

$\& \mathrm{rxb}) *($ one + cxc* $($ one-fxc $))$

$\&-\mathrm{rxa} *((\mathrm{fxn} / \mathrm{fxc}) * * \mathrm{rxa}) *((\mathrm{one}-\mathrm{fxn} / \mathrm{fxc}) * * \mathrm{rxb}) *$

$\&$ (one+cxc* (one-fxc))

$\&+r x b *((f x n / f x c) * *(r x a+o n e)) *((o n e-f x n / f x c)$

$\& * *(\mathrm{rxb}-\mathrm{one})) *($ one $+\mathrm{cxc} *($ one-fxc $))$

$\&-\mathrm{cxc} * \mathrm{fxc} *((\mathrm{fxn} / \mathrm{fxc}) * * \mathrm{rxa}) *(($ one $-\mathrm{fxn} / \mathrm{fxc}) * *$

\& $r x b)$

$\mathrm{dfxndfxc}=r 5 c * d r x g d f x c$

$c$ residual equations on the isv values: $\mathrm{c} 2=\mathrm{c} 20 *($ rate $/$ rate 02$) * * r 2 \mathrm{~m}$

$\mathrm{dc} 2 \mathrm{de}=\mathrm{c} 20 * \mathrm{r} 2 \mathrm{~m} *(($ rate $/$ rate 02$) * *(r 2 \mathrm{~m}-$ one $)) /$ 


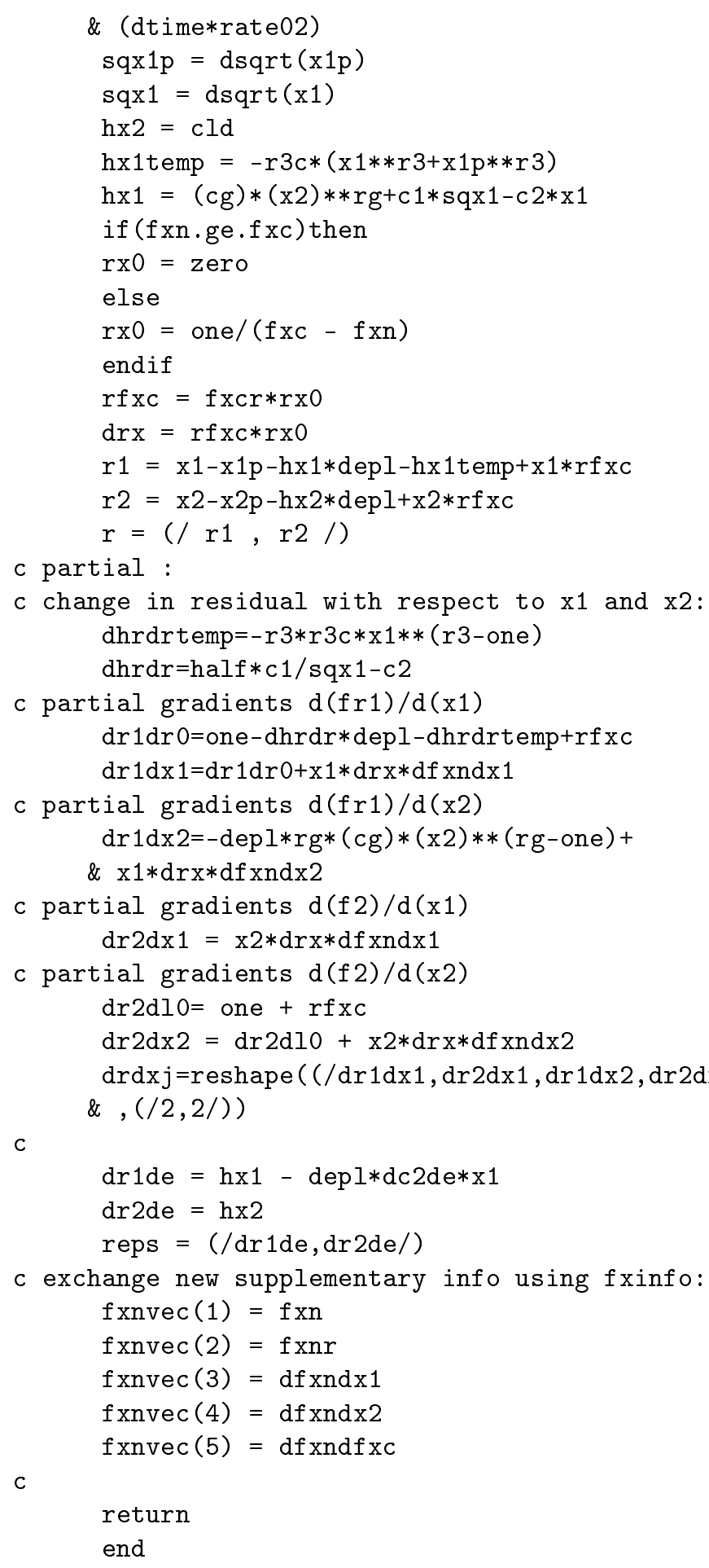

\section{References}

[1] Abaqus $6.11 \quad$ (2011) Abaqus/Standard - Abaqus/Explicit version 6.11. http://www.3ds.com/productsservices/simulia/products/abaqus/

[2] Acharya A, Beaudoin AJ (2000) Grain-size effect in viscoplastic polycrystals at moderate strains. Journal of the Mechanics and Physics of Solids 48(10):2213-2230
[3] Bailey JE, Hirsch PB (1962) The recrystallization process in some polycrystalline metals. Proc.Roy.Soc. A267:11-30

[4] Banerjee B (2007) The Mechanical Threshold Stress model for various tempers of AISI 4340 steel. International Journal of Solids and Structures 44(34):834-859

[5] Baron TJ, Khlopkov K, Pretorius T, Balzani D, Brands D, Schröder J (2016) Modeling of Microstructure Evolution with Dynamic Recrystallization in Finite Element Simulations of Martensitic Steel. Steel research international 87(1):37-45

[6] Beltran O, Huang K, Logé RE (2015) A mean field model of dynamic and post-dynamic recrystallization predicting kinetics, grain size and flow stress. Computational Material Science 102:293-303

[7] Bernacki M, Coupez RLT (2011) Level set framework for the finite-element modelling of recrystallization and grain growth in polycrystalline materials. Scripta Materialia 64:525-528

[8] Brown AA, Bammann DJ (2012) Validation of a model for static and dynamic recrystallization in metals. International Journal of Plasticity 32-33:1735

[9] Cahn JW, Hagel WC (1962) Theory of the pearlite reaction. Decomposition of Austenite by Diffusional Pr) rocesses, 131-196

[10] Chen L, Chen J, Lebensohn R, Ji Y, Heo T, Bhattacharyya S, Chang K, Mathaudhu S, Liu Z, Chen LQ (2015) An integrated fast fourier transformbased phase-field and crystal plasticity approach to model recrystallization of three dimensional polycrystals. Computer Methods in Applied Mechanics and Engineering 285:829-848

[11] Chen SP, Zwaag S, Todd I (2002) Modeling the kinetics of grain-boundary-nucleated recrystallization processes after cold deformation. Metallurgical and Materials Transactions A 33(3):529-537

[12] Dhondt G, Wittig K (1998) CalculiX:A Free Software Three-Dimensional Structural Finite Element Program, http://www.dhondt.de/

[13] Doherty RD, Hughes DA, Humphreys FJ, Jonas JJ, Juul Jensen D, Kassner ME, King WE, McNelley TR, McQueen HJ, Rollett AD (1997) Current issues in recrystallization: A review. Materials Science and Engineering A 238(2):219-274

[14] Électricité de France, Code_Aster, www.codeaster.org/

[15] Estrin Y (1996) Dislocation-density-related constitutive modeling. In: Krausz, A., Krausz, K. (Eds.), Unified constitutive laws of plastic deformation. Academic Press, San Diego, Ch.2, pp 69 - 106 
[16] Estrin Y, Mecking H (1984) A unified phenomenological description of work hardening and creep based on one-parameter models. Acta Metallurgica $32(1): 57-70$

[17] Fan XG, Yang H (2011) Internal-state-variable based self-consistent constitutive modelling for hot working of two-phase titanium alloys coupling microstructure evolution. International Journal of Plasticity 27(11):1833-1852

[18] Follansbee PS, Kocks UF (1998) A constitutive description of copper based on the use of the mechanical threshold stress as an internal state variable. Acta Materialia 36(1):81-93

[19] Galindo-Nava EI, Rivera-Díaz-del-Castillo P (2013) Thermostatistical modelling of hot deformation in FCC metals. International Journal of Plasticity 47:202-221

[20] Goto DM, Garrett RK, Bingert JF, Chen SR, Gray GT (2000) The mechanical threshold stress constitutive-strength model description of HY-100 steel. Metallurgical and Materials Transactions A 31(8):1985-1996

[21] Hallberg H, Ristinmaa M (2013) Microstructure evolution influenced by dislocation density gradients modelled in a reaction-diffusion system. Computational Materials Science 67:373-383

[22] Hallberg H (2013) A modified level set approach to 2D modeling of dynamic recrystallization. Modelling and Simulation in Materials Science and Engineering $21(8)$

[23] Harun A, Holm E, Clode M, Miodownik M, (2006) On computer simulation methods to model Zener pinning. Acta Materialia 54:3261-3273

[24] Humphreys FJ, Hatherly M (1995) Recrystallization and Related Annealing Phenomena, 2nd Edition. Elsevier

[25] Ivasishin O, Vasiliev SSN, Semiatin S (2006) A 3-D Monte-Carlo (Potts) model for recrystallization and grain growth in polycrystalline materials. Material Science and Engineering A 433:216-232.

[26] Jansen van Rensburg GJ (2016) Development and Implementation of State Variable Based User Materials in Computational Plasticity. PhD Thesis, The University of Pretoria, Pretoria, South-Africa

[27] Jansen van Rensburg GJ, Kok S, Wilke DN. (2016) Cyclic effects and recrystallisation in temperature and rate dependent state variable based plasticity. 10th South African Conference on Computational and Applied Mechanics (SACAM 2016), 3-5 October 2016, Potchefstroom, South Africa.

[28] Jansen van Rensburg GJ, Kok S, Wilke DN. (2017) Steel Alloy Hot Roll Simulations and Through-Thickness Variation Using Dislocation
Density-Based Modeling. Metallurgical and Materials Transactions B. DOI :10.1007/s11663-017-10247 .

[29] Ji H, Liu J, Wang B, Fu X, Xiao W, Hu Z (2017) A new method for manufacturing hollow valves via cross wedge rolling and forging: Numerical analysis and experiment validation. Journal of Materials Processing Technology 240:1-11

[30] Kapoor R, Paul B, Raveendra S, Samajdar I, Chakravartty J (2009) Aspects of Dynamic Recrystallization in Cobalt at High Temperatures. Metallurgical and Materials Transactions A 40(4):818-827

[31] Kocks UF (1976) Laws for work-hardening and low temperature creep. Journal of Engineering Materials and Technology 98:76-85

[32] Kocks UF, Mecking H (1979) A mechanism for static and dynamic recovery. In: Haasen, P., Gerold, V., Korstorz, G. (Eds.), Strength of Metals and Alloys. Pergamon, Oxford, pp 345-350

[33] Kocks UF, Mecking H (1979) Physics and phenomenology of strain hardening: the FCC case. Progress in Material Science 48:171-273

[34] Kocks UF, Tomé C, Wenk, H (1998) Texture and Anisotropy. Cambridge University Press

[35] Kok S, Beaudoin AJ, Tortorelli DA (2002) On the development of stage IV hardening using a model based on the mechanical threshold. Acta Materialia 50(7):1653-1667

[36] Lee HW, Im YT (2010) Cellular automata modeling of grain coarsening and refinement during the dynamic recrystallization of pure copper. Material Transactions 51:1614-1620

[37] Lin J, Liu Y, Farrugia DCJ, Zhou M (2005) Development of dislocation-based unified material model for simulating microstructure evolution in multipass hot rolling. Philosophical Magazine 85(18):19671987

[38] Mellbin Y, Hallberg H, Ristinmaa M (2016) Recrystallization and texture evolution during hot rolling of copper, studied by a multiscale model combining crystal plasticity and vertex models. Modelling and Simulation in Materials Science and Engineering $24(7)$

[39] Moelans N, Godfrey A, Zhang Y, Jensen DJ (2013) Phase-field simulation study of the migration of recrystallization boundaries. Physical Review B 88:054103

[40] Montheillet F, Lurdos O, Damamme G (2009) A grain scale approach for modeling steady-state discontinuous dynamic recrystallization. Acta Materialia 57(5):1602-1612 
[41] Mourad HM, Bronkhorst CA, Addessio FL, Cady CM, Brown DW, Chen SR, Gray GT (2014) Incrementally objective implicit integration of hypoelastic-viscoplastic constitutive equations based on the mechanical threshold strength model. Comput Mech 53:941-955

[42] Pietrzyk M (2002) Through-process modelling of microstructure evolution in hot forming of steels. Journal of Materials Processing Technology 125$126: 53-62$

[43] Popova E, Staraselski Y, Brahme A, Mishra R, Inal K (2015) Coupled crystal plasticity-probabilistic cellular automata approach to model dynamic recrystallization in magnesium alloys. International Journal of Plasticity 66:85-102

[44] Riedel H, Svoboda J (2016) A model for strain hardening, recovery, recrystallization and grain growth with applications to forming processes of nickel base alloys. Materials Science and Engineering: A 665:175-183

[45] Roucoules C, Pietrzyk M, Hodgson PD (2003) Analysis of work hardening and recrystallization during the hot working of steel using a statistically based internal variable model. Materials Science and Engineering A 339(1-2):1-9

[46] Simo JC, Hughes TJR (1997) Computational Inelasticity. Springer.

[47] Song JE, McDowell DL (2012) Grain Scale Crystal Plasticity Model with Slip and Microtwinning for a Third Generation Ni-Base Disk Alloy. John Wiley \& Sons, Hoboken, pp 159-166

[48] Sun ZC, Yang H, Han GJ, Fan XG (2010) A numerical model based on internal-state-variable method for the microstructure evolution during hotworking process of TA15 titanium alloy. Materials Science and Engineering A 527(15):3464-3471

[49] Takaki T, Yoshimoto C, Yamanaka A, Tomita Y (2014) Multiscale modeling of hot-working with dynamic recrystallization by coupling microstructure evolution and macroscopic mechanical behavior. International Journal of Plasticity 52:105-116

[50] Tanner AB (1998) Modelling temperature and strain rate history effects in $\mathrm{OFHC} \mathrm{Cu}$. $\mathrm{PhD}$ Thesis, Georgia Institute of Technology

[51] Tanner AB, McDowell DL (1999) Deformation, temperature and strain rate sequence experiments on OFHC $\mathrm{Cu}$. International Journal of Plasticity 15(4):375-399

[52] Varshni YP (1970) Temperature dependence of the elastic constants. Physical Review B 2(10):39523958
[53] Vondrous A, Bienger P, Schreijäg S, Selzer M, Schneider D, Nestler B, Helm D., Mönig R (2015) Combined crystal plasticity and phase-field method for recrystallization in a process chain of sheet metal production. Comput Mech 55:439-452

[54] Weygand D, Brechet Y, Lepinoux J (1998) A vertex dynamics simulation of grain growth in two dimensions. Philosophical Magazine B 78:329-352 